\title{
Correction of real-time satellite precipitation with satellite soil moisture observations
}

\author{
W. Zhan ${ }^{1}$, M. Pan ${ }^{1}$, N. Wanders ${ }^{1,2}$, and E. F. Wood ${ }^{1}$ \\ ${ }^{1}$ Department of Civil and Environmental Engineering, Princeton University, Princeton, NJ, USA \\ ${ }^{2}$ Department of Physical Geography, Utrecht University, Utrecht, the Netherlands \\ Correspondence to: W. Zhan (wzhan@princeton.edu)
}

Received: 28 April 2015 - Published in Hydrol. Earth Syst. Sci. Discuss.: 16 June 2015

Revised: 3 October 2015 - Accepted: 7 October 2015 - Published: 22 October 2015

\begin{abstract}
Rainfall and soil moisture are two key elements in modeling the interactions between the land surface and the atmosphere. Accurate and high-resolution real-time precipitation is crucial for monitoring and predicting the onset of floods, and allows for alert and warning before the impact becomes a disaster. Assimilation of remote sensing data into a flood-forecasting model has the potential to improve monitoring accuracy. Space-borne microwave observations are especially interesting because of their sensitivity to surface soil moisture and its change. In this study, we assimilate satellite soil moisture retrievals using the Variable Infiltration Capacity (VIC) land surface model, and a dynamic assimilation technique, a particle filter, to adjust the Tropical Rainfall Measuring Mission Multi-satellite Precipitation Analysis (TMPA) real-time precipitation estimates. We compare updated precipitation with real-time precipitation before and after adjustment and with NLDAS gauge-radar observations. Results show that satellite soil moisture retrievals provide additional information by correcting errors in rainfall bias. The assimilation is most effective in the correction of medium rainfall under dry to normal surface conditions, while limited/negative improvement is seen over wet/saturated surfaces. On the other hand, high-frequency noises in satellite soil moisture impact the assimilation by increasing rainfall frequency. The noise causes larger uncertainty in the falsealarmed rainfall over wet regions. A threshold of $2 \mathrm{~mm}_{\text {day }}{ }^{-1}$ soil moisture change is identified and applied to the assimilation, which masked out most of the noise.
\end{abstract}

\section{Introduction}

Precipitation is perhaps the most important variable in controlling energy and mass fluxes that dominate climate and particularly the terrestrial hydrological and ecological systems. Precipitation estimates, together with hydrologic models, provide the foundation for understanding the global energy and water cycles (Sorooshian, 2004; Ebert et al., 2007). However, obtaining accurate measurements of precipitation at regional to global scales has always been challenging due to its small-scale, space-time variability, and the sparse networks in many regions. Such limitations impede precise modeling of the hydrologic responses to precipitation. There is a clear need for improved, spatially distributed precipitation estimates to support hydrological modeling applications.

In recent years, remotely sensed satellite precipitation has become a critical data source for a variety of hydrological applications, especially in poorly monitored regions such as sub-Saharan Africa due to its large spatial coverage. To date, a number of fine-scale, satellite-based precipitation estimates are now in operational production. One of the most frequently used is the Tropical Rainfall Measuring Mission Multi-satellite Precipitation Analysis (TMPA) product (Huffman et al., 2007). Over the 17-year lifetime since the launch of the Tropical Rainfall Measuring Mission (TRMM) in 1997 , a series of high-resolution $\left(0.25^{\circ}\right.$ and 3-hourly), quasiglobal $\left(50^{\circ} \mathrm{S}-50^{\circ} \mathrm{N}\right)$, near-real-time, TRMM-based precipitation estimates have been developed and made available to the research and applications communities (Huffman et al., 2007, 2010). Flood forecasting and monitoring is one major application for real-time satellite rainfall products (Wu et al., 2014). However, the applicability of satellite precipitation products for near-real-time hydrological applications that in- 
clude drought and flood monitoring has been hampered by their need for gauge-based adjustment.

While it is possible to create such estimates solely from one type of sensor, researchers have increasingly moved to using combinations of sensors in an attempt to improve accuracy, coverage and resolution. A promising avenue for rainfall correction is through the assimilation of satellite-based surface soil moisture into a water balance model (Pan and Wood, 2006). Over land, the physical relationship between variations in soil water storage and rainfall accumulation contain complementary information that can be exploited for the mutual benefit of both types of products (Massari et al., 2014; Crow et al., 2009). Unlike instantaneous rain rate, satellite surface soil moisture retrievals utilize low-frequency microwave signals and possess some memory reflecting antecedent rainfall amounts.

Studies have demonstrated that in situ (Brocca et al., 2009, 2013; Matgen et al., 2012) and satellite (Francois et al., 2003; Pellarin et al., 2008, 2013; Brocca et al., 2014) estimates of surface soil moisture could contribute to precipitation estimates by providing useful information concerning the sign and magnitude of antecedent rainfall accumulation errors. In particular, Brocca et al. (2014) estimated daily rainfall on a global scale based on satellite SM products by inverting the soil water balance equation. Crow et al. (2003, 2009, 2011) corrected space-borne rainfall retrievals by assimilating remotely sensed surface soil moisture retrievals into an Antecedent Precipitation Index (API) based soil water balance model using a Kalman filter (Kalman, 1960). However, these studies focused on multi-day aggregation periods and a space aggregated correction at $1^{\circ}$ resolution for the corrected precipitation totals. This limits their applicability in applications such as near real-time flood forecasting. Wanders et al. (2015) tried to overcome this limitation by the correction of 3-hourly satellite precipitation totals with a set of satellite soil moisture and land surface temperature observations. One important conclusion by Wanders et al. (2015) is that their results showed the limited potential for satellite soil moisture observations for correcting precipitation at high resolution if "all-weather" - i.e., microwave-based - land surface temperatures are not available coincidently, as was the case with AMSR-E.

But this is not always the case, and it is also noted that current low-frequency microwave soil moisture missions (specifically SMAP and SMOS) do not have radiometers at frequencies useful for estimating land surface temperatures, even though a $37 \mathrm{GHz}$ sensor is part of the AMSR2 system. In fact, SMAP and SMOS use LST from weather model analysis fields in their algorithms. Unfortunately, the lowest microwave frequency of AMSR2 precludes retrieving soil moisture from many areas with heavy vegetation, and AMSR2 has a significant dry bias with less availability than AMSR-E, but is no longer operable. So, improvements to satellite precipitation from the Global Precipitation Mission products must rely solely on satellite soil moisture products, and the improvements to the assimilation algorithms are the goal of this study.

Thus, we focus exclusively on the usefulness of assimilating soil moisture products to improve satellite rainfall. We propose as part of the work how to improve the generation of rain particles and the bias correction of the satellite soil moisture observations, as well as to enhance the assimilation algorithm to maximize the information that can be gained from using soil moisture alone to adjust precipitation. Due to the very strong and complicated spatial structure of precipitation, that is non-Gaussian and non-stationary in both time and space (Wanders et al., 2015), a more advanced method is applied to generate possible precipitation fields than were used in earlier studies or in Wanders et al. (2015) (see Sect. 2.2.2). Furthermore, a more advanced bias-correction method is also applied to account for the reported problems in the second-order statistics of the soil moisture retrievals. We used a soil moisture remote sensing product to improve the real-time remote sensing precipitation product, TMPA 3B42RT, through a particle filter (PF), and therefore offer an improved basis for quantitatively monitoring and predicting flood events, especially in those parts of the world where in situ networks are too sparse to support more traditional methods of hydrologic monitoring and prediction. The precipitation enhancement experiments are carried out over the continental US (CONUS) and the precipitation skill is validated against the NLDAS gauge-radar precipitation product. Section 5 presents a comparison of the results from this study to the earlier studies related to improving satellite precipitation.

\section{Methods}

\subsection{Overview}

Random replicates of satellite precipitation are generated based on real-time TMPA (3B42RT) retrievals and its uncertainty (Pan et al., 2010), which are then used to force the VIC land surface model (LSM) where one output of interest is surface soil moisture. Satellite soil moisture data products are compared and merged with the 3B42RT product to improve the accuracy of the satellite precipitation estimates. A schematic for the study approach is provided in Fig. 1. Based on real-time 3B42RT retrievals, a set of possible precipitation estimates (a.k.a. replicates or particles) $\left\{p^{i} t\right\}_{i=1,2, \ldots, N}$ is generated with assigned initial prior probability weights $\left\{w^{i}\right\}_{i=1,2, \ldots, N}$. These rainfall rates are then used to force the VIC land surface model to produce soil moisture predictions $\left\{\theta^{i}\right\}_{i=1,2, \ldots, N}$. Retrievals of AMSR-E satellite surface soil moisture using the Land Surface Microwave Model (LSMEM) (Pan et al., 2014) are then merged with the LSM-based soil moisture within the particle filter (PF) that compares AMSR-E/LSMEM changes in soil moisture, $\Delta \mathrm{SM}$, to the LSM predicted soil moisture 
Table 1. Error statistics of recovered precipitation and effect of surface saturation in the idealized experiment $\left(\mathrm{mm} \mathrm{day}^{-1}\right)$.

\begin{tabular}{|c|c|c|c|c|c|c|c|c|c|c|c|c|c|c|c|}
\hline $\begin{array}{l}\text { [Recovered } \\
\text { NLDAS] - } \\
{[\text { NLDAS] }}\end{array}$ & $\begin{array}{l}\text { [3B42RT] - } \\
{[\mathrm{NLDAS}]}\end{array}$ & 0 & $0-0.2$ & $0.2-0.5$ & $0.5-1.0$ & $1.0-1.5$ & $1.5-2$ & $2-2.5$ & $2.5-5.0$ & $5.0-7.5$ & $7.5-10$ & $10-15$ & $15-20$ & $20-25$ & $>25$ \\
\hline \multirow{2}{*}{$\begin{array}{l}\text { All surface } \\
\text { conditions }\end{array}$} & Bias & 0.24 & 0.20 & 0.37 & 0.51 & 0.71 & 0.87 & 1.09 & 0.67 & 1.16 & 1.30 & 2.51 & 3.32 & 3.75 & 3.95 \\
\hline & MAE & 0.40 & 0.42 & 0.66 & 0.86 & 1.14 & 1.41 & 1.70 & 1.48 & 2.24 & 2.63 & 4.21 & 5.56 & 6.70 & 9.76 \\
\hline \multirow{2}{*}{$\begin{array}{l}\text { Unsaturated } \\
\text { surface }\end{array}$} & Bias & 0.23 & 0.19 & 0.29 & 0.40 & 0.52 & 0.68 & 0.82 & 0.65 & 1.10 & 1.27 & 2.19 & 2.88 & 3.14 & 3.14 \\
\hline & MAE & 0.39 & 0.41 & 0.59 & 0.75 & 0.95 & 1.21 & 1.43 & 1.45 & 2.17 & 2.58 & 3.88 & 5.11 & 6.07 & 8.94 \\
\hline \multirow{2}{*}{$\begin{array}{l}\text { Saturated } \\
\text { surface }\end{array}$} & Bias & 2.31 & 5.06 & 47.65 & 42.58 & 50.67 & 44.09 & 59.64 & 6.83 & 16.09 & 9.19 & 46.47 & 57.98 & 65.33 & 64.09 \\
\hline & MAE & 3.35 & 5.54 & 48.71 & 43.73 & 52.43 & 46.96 & 61.85 & 9.64 & 21.42 & 15.01 & 49.07 & 60.78 & 69.53 & 70.73 \\
\hline
\end{tabular}

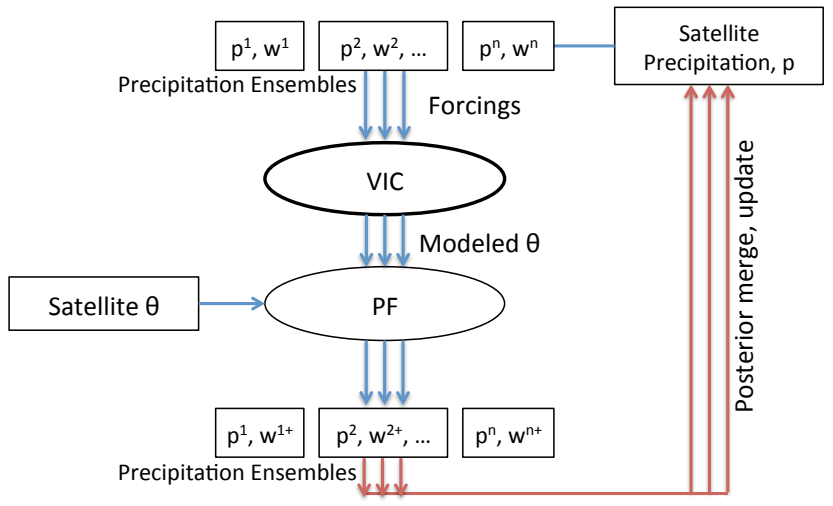

Figure 1. Schematic for the dynamic assimilation of AMSRE/LSMEM $\triangle \mathrm{SM}$ into TMPA (3B42RT) with the particle filter (PF).

changes. From these, posterior weights $\left\{w^{i+}\right\}_{i=1,2, \ldots, N}$ are calculated for each precipitation member (particle) that takes into account the uncertainties of AMSR-E/LSMEM $\triangle \mathrm{SM}$ retrievals. From these updated weights, an updated precipitation probability distribution is constructed, where the precipitation particle with the highest probability is taken as the "best" adjusted precipitation estimate (3B42RT $\left.\mathrm{ADJ}_{\mathrm{AJ}}\right)$. The procedure is carried out over the continental US (CONUS) region on a grid-by-grid basis $\left(0.25^{\circ}\right)$ and a daily time step. Allowing for a 6-month model spin-up period, the adjustment is done from January 2003 to July 2007.

\subsection{Modeling, statistical tools and data sources}

\subsubsection{The particle filter}

Data assimilation methods are capable of dynamically merging predictions from a state equation (i.e., the land surface model) with measurements (i.e., AMSR-E retrievals) to minimize uncertainties from both the predictions and measurements. It is assumed that the source of uncertainty in the land surface model predictions comes solely from the real-time satellite precipitation, so that the particle filter $(\mathrm{PF})$ provides an algorithm to update the precipitation based on the AMSR$E$ retrievals. The state evolution of a particle filter from discrete time $t-1$ to $t$ can be represented as $\theta_{t}=f_{t}\left(\theta_{t-1}, p_{t}, \kappa_{t}, \alpha_{t}\right)$

where $\theta_{t}$ is the first-layer soil moisture at time $t$, whose value is predicted by the state equation (Eq. 1) as $f_{t}(\bullet)$, and in the study is the hydrological model VIC, which takes in forcing data, including precipitation $\left(p_{t}\right)$ and other forcings $\left(\kappa_{t}\right)$; and simulates land surface states (soil moisture and soil temperatures at various levels, snow, etc.) and fluxes (evapotranspiration, runoff) at time $t$. Herein we are basically interested only in the first-layer (top $10 \mathrm{~cm}$ ) soil moisture state and precipitation forcing, so other states and fluxes are not explicitly shown. $\alpha_{t}$ is the random error in the prediction of $\theta_{t}$, whose statistics are known but not its value at any specific time.

At time $t$, the satellite surface soil moisture retrieval, $\theta_{t}^{*}$, can be related to the VIC modeled first-layer soil moisture $\theta_{t}$ as

$\theta_{t}^{*}=h_{t}\left(\theta_{t}, \beta_{t}\right)$,

where $h_{t}$ is taken as a regression that transforms the VIC simulated first-layer soil moisture to satellite surface soil moisture. $\beta_{t}$ is the noise in this regression relationship. The two noises $\alpha_{t}$ and $\beta_{t}$ are assumed to be independent of each other at all times $t$.

At time $t$, given a 3B42RT precipitation estimate, $p_{t}^{\text {sat }}$, a set of N precipitation replicates $\left\{p_{t}^{i}\right\}_{i=1,2, \ldots, N}$ and their associated initial prior probability weight $\left\{w_{t}^{i}\right\}_{i=1,2, \ldots, N}$ are generated.

$g\left(p_{t}^{\mathrm{sat}}\right) \sim\left\{p_{t}^{i}, w_{t}^{i}\right\}_{i=1,2, \ldots, N}$

$\sum_{i=1}^{N} w_{t}^{i}=1$

$g()$ is a probability density function. For $N$ precipitation replicates, $\left\{p_{t}^{i}\right\}_{i=1,2, \ldots, N}$, the propagation of the states from time step $(t-1)$ to $t$ is by the VIC land surface model represented in Eq. (1). The VIC land surface model simulates the $10 \mathrm{~cm}$ first-layer soil moisture, $\left\{\theta_{t}^{i}\right\}_{i=1,2, \ldots, N}$, for each precipitation replicate: 

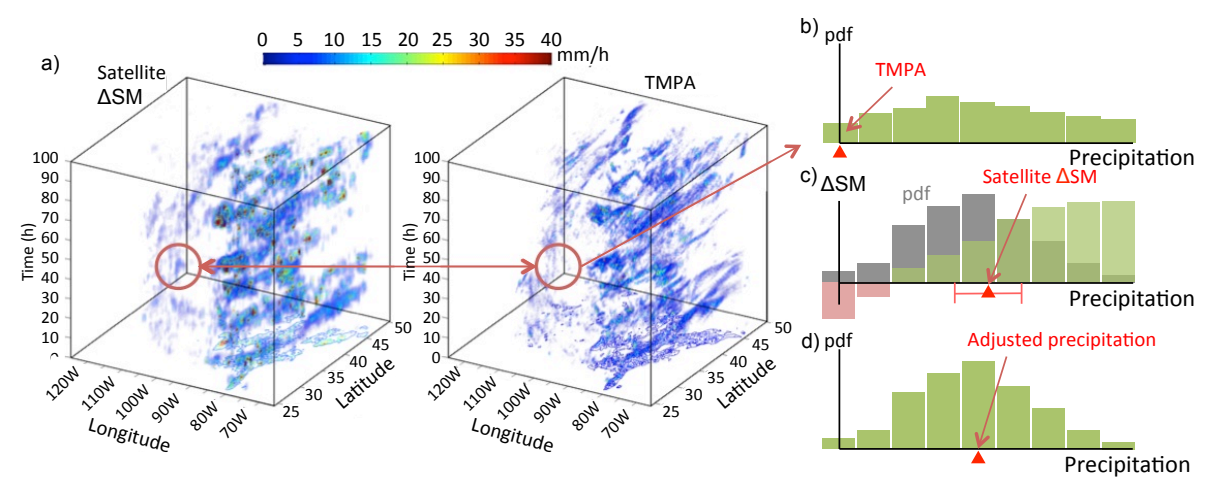

Figure 2. Schematic for the strategy for processing prior and posterior probability densities in the particle filter. The missing rainfall event in TMPA (circled in the right panel of a, correspond to red triangle in b) against satellite signals as detected by AMSR-E/LSMEM $\triangle$ SM (circled in the left panel of a, correspond to red triangle in $\mathbf{c}$ ), and recovered by assimilating AMSR-E/LSMEM $\triangle$ SM into TMPA (marked by red triangle in $\mathbf{d}$ ).

$\left\{\theta_{t}^{i}=f_{t}\left(\theta_{t-1}, p_{t}^{i}, \kappa_{t}, \alpha_{t}\right)\right\}_{i=1,2, \ldots, N}$

with the associated weights assigned to the precipitation member:

$$
\left\{\theta_{t}^{i}, w_{t}^{i}\right\}_{i=1,2, \ldots, N}=\left\{f_{t}\left(\theta_{t-1}, p_{t}^{i}, \kappa_{t}, \alpha_{t}\right), w_{t}^{i}\right\}_{i=1,2, \ldots, N} .
$$

If the satellite soil moisture retrieval at time $t$ is $\theta_{t}^{*}$, the update of precipitation forcing is accomplished by updating the importance weight of each replicate given the "measurement" $\theta_{t}^{*}:$

$$
\begin{aligned}
& w_{t}^{i+} \sim\left\{g\left(\theta_{t}^{i} \mid \theta_{t}^{*}\right)\right\}_{i=1,2, \ldots, N} \\
& \sum_{i=1}^{N} w_{t}^{i+}=1 .
\end{aligned}
$$

The likelihood function $g\left(\theta_{t}^{i} \mid \theta_{t}^{*}\right)$ can be derived from $h_{t}$ and $g\left(\beta_{t}\right)$. The schematic of the utilized strategy is shown in Fig. 2 with a synthetic example of a missing rainfall pattern in the TMPA compared with satellite $\Delta$ SM. The primary disadvantage of the particle filter is the large number of replicates required to accurately represent the conditional probability densities of $p_{t}$ and $\theta_{t}$. When the measurements exceed a few hundred, the particle filter is not computationally practical for land surface problems. Considering computation efficiency, we set the number of independent particles, $N$, from the prior distribution to be 200 .

\subsubsection{Precipitation replicates generation}

We generate precipitation replicates, $\left\{p_{t}^{i}\right\}_{i=1,2, \ldots, N}$, based on statistics comparing NLDAS and 3B42RT precipitation, as shown in Fig. 3. Given a 3B42RT precipitation measurement (binned by magnitude), with bin minimum and maximum indicated in Fig. 3, precipitation replicates are generated based on the corresponding 15th, 30th, 70th, 85th percentiles and

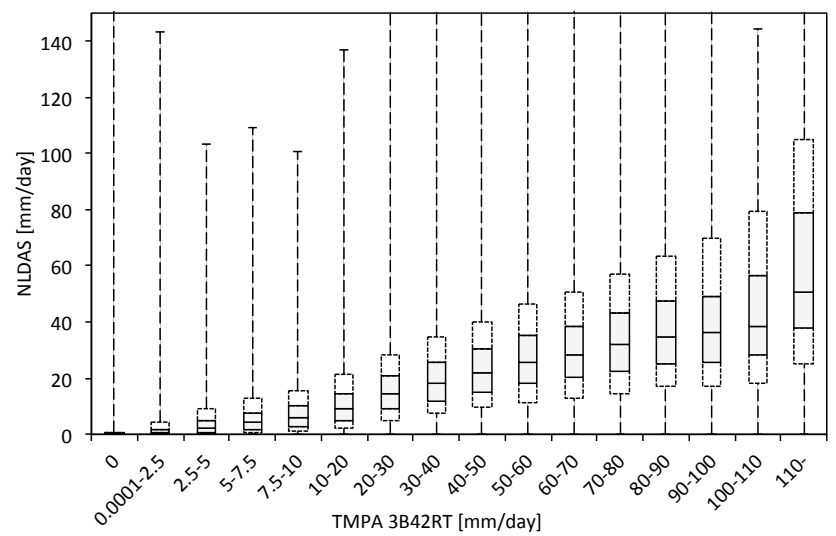

Figure 3. Statistics of NLDAS precipitation given 3B42RT precipitation measurement. Boxplot shows the minimum, $15 \%$ quantile, $30 \%$ quantile, median, $70 \%$ quantile, $85 \%$ quantile and maximum value of NLDAS precipitation given 3B42RT precipitation in a certain bin.

the maximum NLDAS precipitation of the particular quantile bin as follows: $15 \%$ of the replicates are generated with values uniformly distributed from 0 and 15 th percentile; $15 \%$ of replicates with values from 15 th to 30 th percentile; $20 \%$ of replicates with values from 30th percentile to the median; $20 \%$ of the replicates generated from the median to 70th; $15 \%$ with values from 70 th to 85 th percentile; and $15 \%$ from the 85 th percentile to the maximum precipitation value. Note that although the generation of particles is based on statistics calculated from NLDAS, results show little difference generating precipitation ensembles uniformly distributed between 0 and $200 \mathrm{~mm} \mathrm{day}^{-1}$.

\subsubsection{AMSR-E/LSMEM soil moisture retrievals}

The soil moisture product is derived from multiple microwave channels of the Advanced Microwave Scanning Ra- 
diometer for EOS (AMSR-E) instrument. The retrieval algorithm by Pan et al. (2014) is an enhanced version of the Land Surface Microwave Emission Model (LSMEM). The near surface soil moisture and vegetation optical depth (VOD) are estimated simultaneously from a dual polarization approach that utilizes both horizontal $(\mathrm{H})$ and vertical $(\mathrm{V})$ polarizations measurement by the space-borne sensor. The input AMSRE brightness temperature comes from the NSIDC AMSRE/Aqua Daily Global Quarter-Degree Gridded Brightness Temperatures product (overlapping swaths in the same day are truncated so that only the latest one is present). Consequently, the soil moisture retrievals are also gridded at $0.25^{\circ}$ with one ascending map and one descending map at the daily time step. A maximum threshold value of $0.6 \mathrm{~m}^{3} \mathrm{~m}^{-3}$ has been applied manually to reduce error from open water bodies. According to Pan et al. (2014), the soil moisture data set based on observations from AMSR-E are shown to be consistent at large scales in terms of reproducing the spatial pattern of soil moisture from VIC land surface model simulation. Ascending soil moisture retrievals (equatorial crossing time 01:30 LT - local time) is assimilated in this study.

Similarly, while the spatial patterns of the basic statistics of AMSR-E/LSMEM SM retrievals compare well to VIC simulations (Pan et al., 2014), VIC has its top layer $(10 \mathrm{~cm})$, which is deeper than the detection depth of AMSR-E, so that the mean and temporal variability of the retrievals are higher than the VIC simulated soil moisture (Fig. 4 in Pan et al., 2014). Considering this difference between detection depths, we pre-process soil moisture retrievals for each pixel as follows:

1. Rescale soil moisture retrievals (AMSR-E/LSMEM $\mathrm{SM})$ to have the same minimum and maximum range as VIC-simulated first-layer soil moisture.

2. Calculate a daily soil moisture change. As satellite retrievals are manually truncated to be no more than $0.6 \mathrm{~m}^{3} \mathrm{~m}^{-3}$ (equivalent to $60 \mathrm{~mm}$ of water in the top soil layer in VIC), retrievals larger than $0.6 \mathrm{~m}^{3} \mathrm{~m}^{-3}$ are excluded.

3. Fit a second-order polynomial regression model with $\Delta \mathrm{SM}$ (all units in $\mathrm{mm}$ of water in the top layer) from satellite and VIC simulation on a monthly basis and $3 \times 3$ grid scale (window).

After pre-processing, the distribution of soil moisture change matches fairly well with $\Delta \mathrm{SM}_{\mathrm{VIC}}$ (Fig. 4). The mean absolute difference reduces from a spatial average of 5.25 to $0.71 \mathrm{~mm} \mathrm{day}^{-1}$, with relatively larger value over the eastern CONUS. According to Pan et al. (2014), the no-skill or negative-skill areas occur mostly over eastern dense forests due to vegetation blockage of the soil moisture signal (Pan et al., 2014). The accuracy of soil moisture retrievals is also limited by mountainous topography and the occurrence of snow and frozen ground during winter whose identification

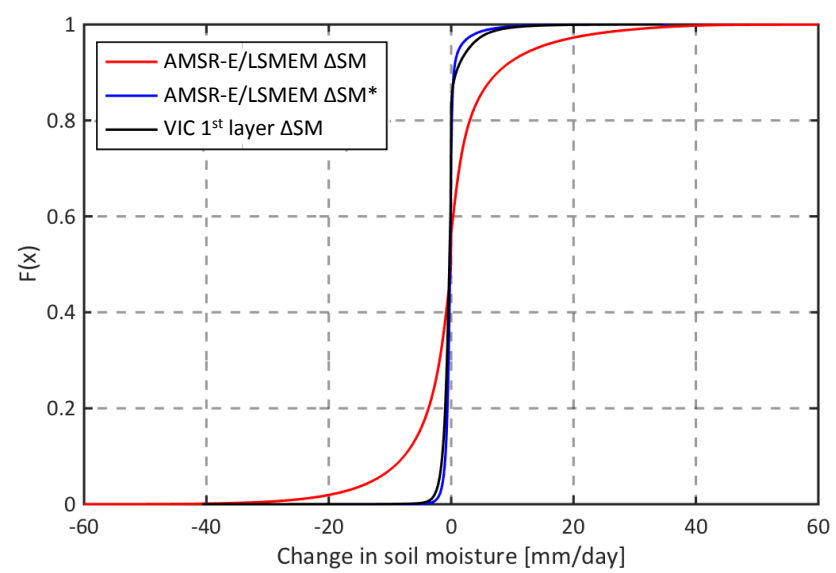

Figure 4. Empirical cumulative distribution function of changes in soil moisture from top layer soil moisture from NLDAS precipitation forced VIC simulation (black), and AMSR-E/LSMEM soil moisture retrieval before (red) and after (blue) pre-processing.

from satellite observations is often difficult. For the purpose of this study, we assign zero weight to the $3 \mathrm{~B} 42 \mathrm{RT}_{\mathrm{ADJ}}$ and rely exclusively on the initial 3B42RT precipitation for time steps when the VIC model predicts snow cover or frozen surfaces.

\subsubsection{VIC land surface model}

The Variable Infiltration Capacity (VIC) model (Liang et al., 1994) is used to dynamically simulate the hydrological responses of soil moisture to precipitation, surface radiation and surface meteorology. The VIC model solves the full energy and water balance over each $0.25^{\circ}$ grid cell independently, thus ensuring its computational efficiency. The assumption of independency poses limitation on the application of LSM at very high spatial resolution (e.g., $1 \mathrm{~km} \times 1 \mathrm{~km})$ over large areas. Three-layer soil moisture is simulated through a soil-vegetation-atmosphere transfer (SVAT) scheme, which also accounts for sub-grid-scale heterogeneity of vegetation, soil and topography. A detailed soil moisture algorithm description can be found in Liang et al. (1996). The VIC model has been validated extensively over CONUS by evaluating soil moisture and simulations to observations (Robock et al., 2003; Schaake et al., 2004).

\section{Idealized experiment}

Before applying the particle filter assimilation algorithm to 3B42RT precipitation estimates, we conducted an idealized experiment where we treat the NLDAS precipitation as the "truth" and the NLDAS precipitation forced VIC simulations as "satellite observed" soil moisture. As an idealized experiment, we adjust TMPA real-time precipitation estimates based on these "satellite observations". Phase 2 of the North American Land Data Assimilation System (NLDAS-2) rain- 

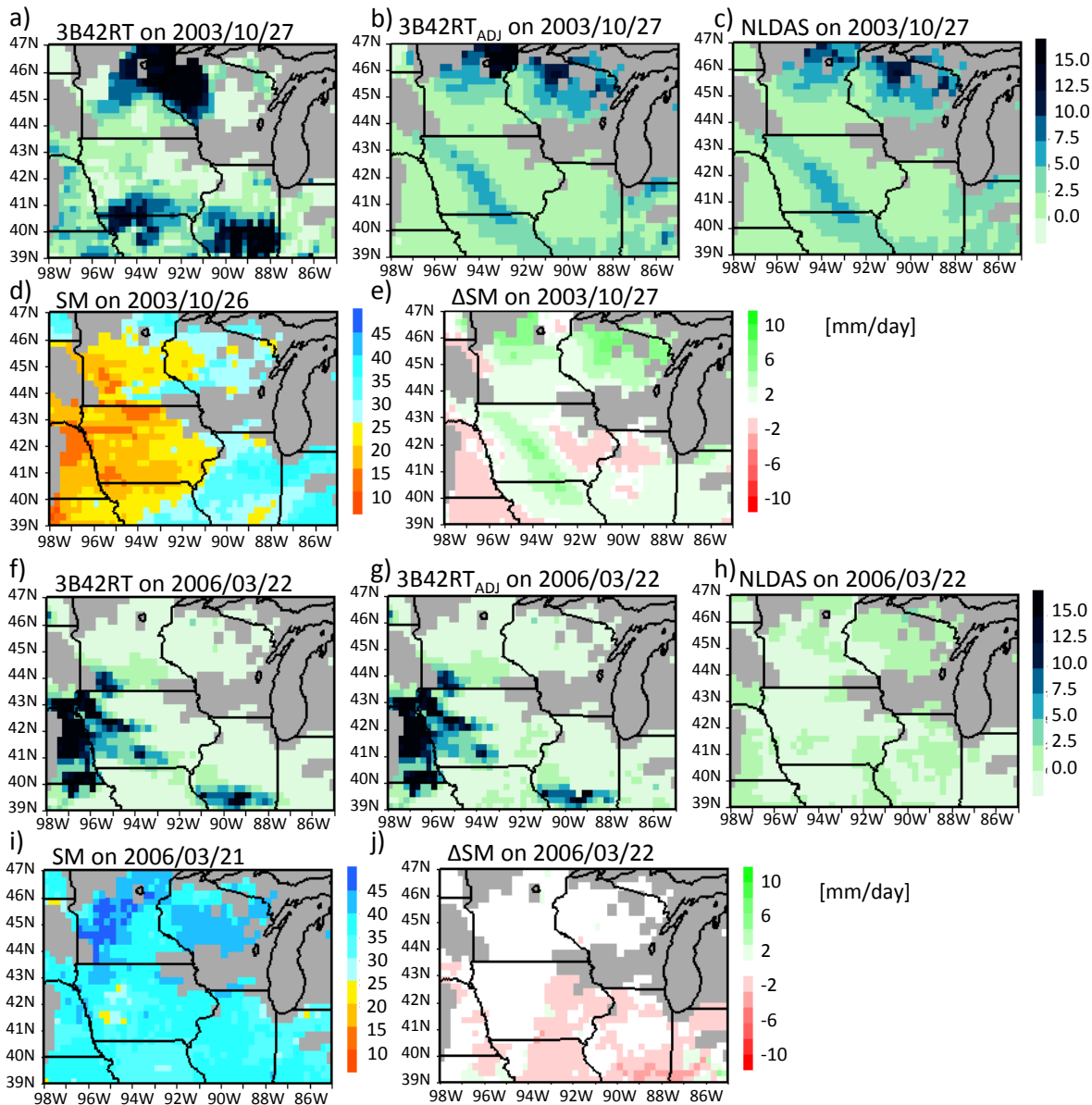

Figure 5. Two cases with recovered spatial rainfall pattern in the idealized experiment after merging satellite soil moisture retrieval on (a)-(e) 27 October 2003 and (f)-(j) 22 March 2006.

fall forcing combines hourly WSR-88D radar analyses from the National Weather Service (NWS) and daily gauge reports $(\sim 13000$ /day) from the Climate Prediction Center (CPC) (Ek et al., 2011). The data set, with a spatial resolution of $0.125^{\circ}$ and hourly observations, was pre-processed into $0.25^{\circ}$ daily precipitation to be consistent with that of 3B42RT and AMSR-E/LSMEM SM data sets. Hourly NLDAS and 3-hourly 3B42RT precipitation is aggregated into daily precipitation defined by a period shifted $\sim 7.5 \mathrm{~h}$ into the future (21:00-21:00 LT), allowing for a necessary delay for soil moisture to respond to incoming rainfall. The idealized experiment is designed to test whether the algorithm is able to retrieve rainfall forcing with soil moisture change, assuming that the soil moisture observations are $100 \%$ accurate.

Results show that, with the knowledge of first-layer soil moisture change (via the "satellite observations"), the adjustment is able to recover intensity and spatial pattern of forcing precipitation (Fig. 5g). Average mean absolute error (MAE) of daily rainfall amount is reduced by $52.9 \%$ (2.91 to $1.37 \mathrm{~mm} \mathrm{day}^{-1}$ ) over the region. Figure $5 \mathrm{a}$ to e shows an example of the recovered rainfall field from the idealized ex- periment for 27 October 2003. The spatial pattern matches the original NLDAS precipitation well.

\subsection{Effect of surface soil saturation}

While successfully recovering the general pattern of NLDAS precipitation based on first-layer soil moisture, the idealized experiment is not always able to recover the precipitation volume due to the fact that the top-layer soil moisture alone does not contain the complete memory of the previous day's rainfall. Deeper soil moisture, evapotranspiration and runoff also carry part of this information. As the surface gets wetter, the VIC first-layer soil moisture has smaller variation. If the incoming precipitation brings the surface to saturation, the VIC model redistributes the soil moisture vertically though vertical moisture flow and generates runoff. Hence, soil moisture increments, $\Delta S M$, near saturation are less correlated with incoming precipitation as they change minimally to additional incoming rainfall. An example demonstrating this saturation effect is shown in Fig. $5 \mathrm{f}$ to $\mathrm{j}$. When incoming precipitation brings the surface SM to (near) saturation, there is very lim- 
ited improvement after the adjustment. Because of the low sensitivity of the soil surface to precipitation, there is little change in $\triangle \mathrm{SM}$ in response to precipitation variations among the replicates. It is almost always the case that the algorithm is not able to find a "matching" $\Delta \mathrm{SM}$.

We separately evaluate the skill improvement in the recovered NLDAS precipitation with and without surface saturation. Figure 6 (statistics provided in Table 1) confirms the effect of surface saturation on adjusted precipitation, which is well described in previous studies (e.g., Brocca et al., 2013, 2014). The recovered precipitation, when the surface soil is saturated, only contributes more noise rather than an improvement to the rainfall estimates. The VIC model computes the moisture flow between soil layers using an hourly time step. If the first-layer soil moisture exceeds its maximum capacity, it is considered to be a surface saturation case. As seen in Fig. 5, there is very limited or negative skill in the recovered precipitation under saturated surface soil moisture conditions. Such circumstances are identified and the AMSR-E/LSMEM $\triangle$ SM observation disregarded by assigning zero weight to the $3 \mathrm{~B} 42 \mathrm{RT}_{\mathrm{ADJ}}$ values. Thus, for wetter areas with heavy precipitation that potentially would bring the surface soil moisture to saturation, the 3B42RT product is less likely to be adjusted according to satellite $\Delta \mathrm{SM}$, and the best precipitation estimate is 3B42RT.

\subsection{Effect of SM uncertainty}

In the idealized experiment, NLDAS-VIC soil moisture is taken as truth with zero uncertainty associated with $\left(\theta_{t}^{*}\right)$. However, this assumption is not valid for real satellite SM retrievals, mean absolute error of which is approximately $3 \%$ vol. vol. ${ }^{-1}$ (McCabe et al., 2005). To consider this, we added error to the "truth" SM (normally distributed with zero mean and standard deviations of 1, 2, 3, 4 and $5 \mathrm{~mm}$ ), and simulated the effect of SM uncertainty to evaluate the associated adjustment errors. Figure 7 shows that larger soil moisture observation errors lead to larger error variation after adjustment. This also suggests that soil moisture responds to precipitation non-linearly based on different initial conditions. An estimated wetter surface has lower sensitivity to an incoming rainfall amount, resulting in larger error in the recovered NLDAS precipitation. As shown in Fig. 7, the error standard deviation of the recovered NLDAS precipitation increases with surface water content (statistics shown in Table 2). As we add noise larger than $N(0.1 \mathrm{~mm})$ into "true" SM observation, there is a wet bias of approximately $1 \mathrm{~mm} \mathrm{day}^{-1}$ regardless of first-layer soil moisture level. This suggests that when the difference between first-layer SM and saturation is less than $8 \mathrm{~mm}$, the median of the errors in the recovered NLDAS precipitation grows from 0.16 to $1.89 \mathrm{~mm} \mathrm{day}^{-1}$ when we add $N(0.5 \mathrm{~mm})$ noise, while the inter-quantile range (IQR) increases from 1.71 to $7.04 \mathrm{~mm} \mathrm{day}^{-1}$. Acknowledging such a wet bias, to avoid introducing any more unintentional bias
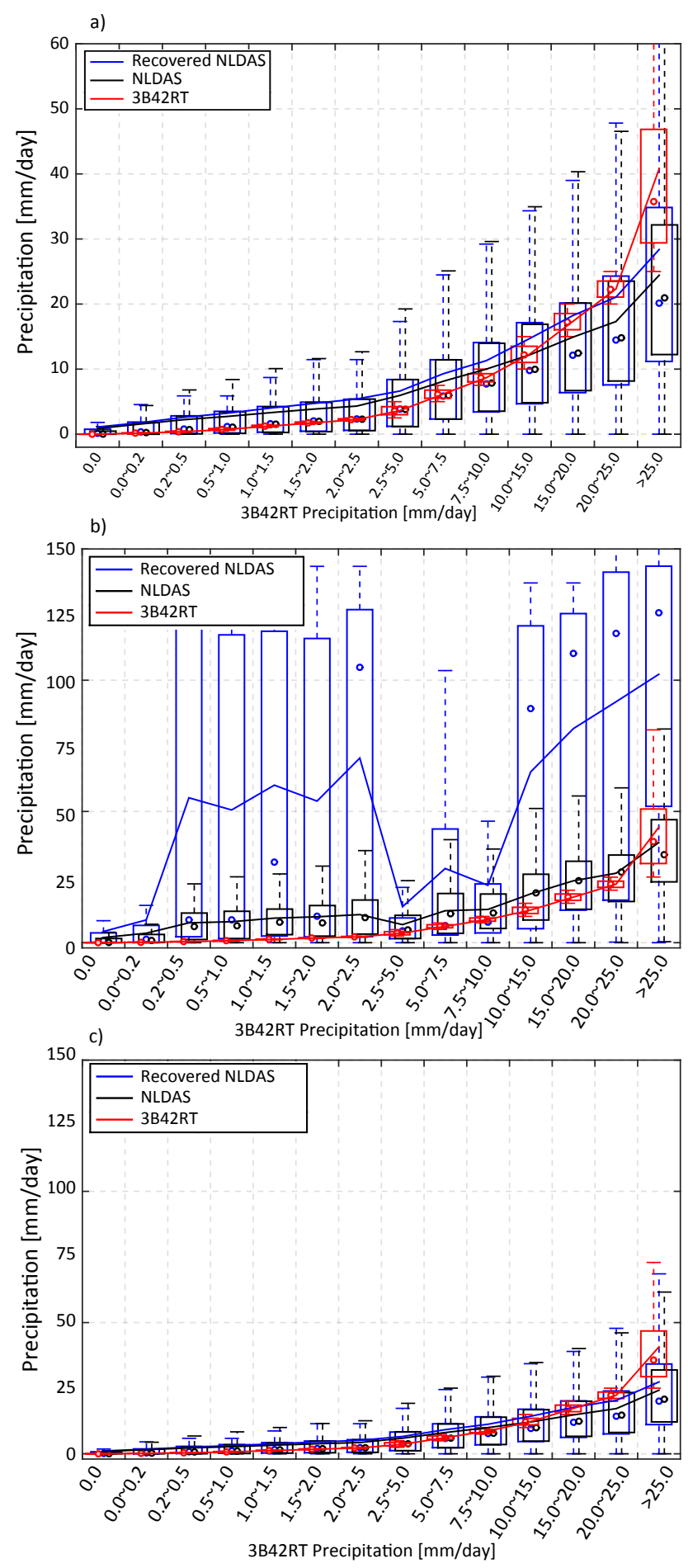

Figure 6. Accuracy of recovered precipitation in an idealized experiment: (a) overall performance and separately comparing the improvement performance of recovered NLDAS precipitation (b) with and (c) without surface saturation condition. Statistics provided in Table 1. 
in the $3 \mathrm{~B} 42 \mathrm{RT} \mathrm{ADJ}_{\mathrm{AD}}$ estimates, we take as zero the uncertainty of AMSR-E/LSMEM SM retrievals, i.e., we take $h_{t}\left(\theta_{t}\right)$ as our single observation $\theta_{t}^{*}$ and adjust the 3B42RT estimates accordingly.

It is noteworthy that the soil moisture change is calculated based on previous days' soil water contents. Therefore errors tend to accumulate over time until they are "re-set" when a significant precipitation event takes place. This type of uncertainty accounts for a small portion of the total error in the adjusted precipitation (black being the no-error case in Fig. 7 with the "true" change in soil moisture from every time step). As complete global coverage is not provided with each orbit of the AMSR-E sensor, on average $44.01 \%$ of the time steps $\left(<0.6 \mathrm{~m}^{3} \mathrm{~m}^{-3}\right)$ during the study period have observations, with more frequent overpasses at higher latitudes (Fig. 4e in Pan et al., 2014). This observation gap unavoidably introduces extra uncertainty into the retrieval of the precipitation signal. To further avoid possible additional errors, we update the forcing rainfall when a $\Delta \mathrm{SM}$ temporal match $( \pm 0.4 \mathrm{~mm})$ is available, and keep the original precipitation if a match is not available.

\section{Improvement on real-time precipitation estimates and their validation}

The adjustment of real TMPA 3B42RT retrievals based on AMSR-E/LSMEM $\triangle \mathrm{SM}$ is carried out using the methods described in Sect. 2.2.3, and results from the idealized experiment (Sect. 3) with regard to the circumstances where an adjustment is applied.

An example of TMPA 3B42RT adjustment is provided in Fig. 8, where a snapshot of the rainfall field is shown (Fig. 8b) and compared with NLDAS on 26 May 2006 and the adjusted rainfall pattern based on AMSR-E/LSMEM $\triangle \mathrm{SM}$. The $3 \mathrm{~B}_{42} \mathrm{RT}_{\mathrm{ADJ}}$ rainfall field (Fig. 8c) is similar in terms of its spatial distribution compared to NLDAS precipitation estimates (Fig. 8d).

On average, TMPA 3B42RT and AMSR-E/LSMEM $\Delta$ SM have a spatial Pearson correlation coefficient of 0.37 (shown in Fig. 9, left panels), compared to 0.52 for the correlation between NLDAS and $\triangle \mathrm{SM}$. After the adjustment procedure, the Pearson correlation coefficient between $3 \mathrm{~B} 42 \mathrm{RT}_{\mathrm{ADJ}}$ and AMSR-E/LSMEM $\triangle$ SM increases to 0.53 (shown in Fig. 9), indicating that the correction method is successful. A belowaverage increase in correlation is found over the western mountainous region, the Great Lakes region and the eastern high vegetated and populated region. Additionally, the satellite soil moisture suffers from snow/ice/standing water contamination, which affects the potential for improved results after correction. The $3 \mathrm{~B} 42 \mathrm{RT}_{\mathrm{ADJ}}$ has significant improvement over 3B42RT in terms of long-term precipitation bias. The bias in 3B42RT annual mean precipitation is reduced by $20.6 \%$, from $-9.32 \mathrm{~mm} \mathrm{month}^{-1}$ spatial average in $3 \mathrm{~B} 42 \mathrm{RT}$ to $-7.40 \mathrm{~mm}^{\text {month }}{ }^{-1}$ in $3 \mathrm{~B} 42 \mathrm{RT} \mathrm{ADJ}_{\mathrm{AJ}}$ (shown in Fig. 9, right panels). Frequency of rain days generally increases significantly everywhere (Fig. 10). The NLDAS data (Fig. 10, right panels) suggest an almost constant drizzling rainfall over parts of the western mountainous area (Montana, Idaho, Wyoming and Colorado), while assimilating AMSR-E/LSMEM $\triangle \mathrm{SM}$ data sets do not have a signal of higher rainfall frequency (Fig. 10, middle panels). This is possibly due to deficiencies in satellite retrievals over the mountainous areas and frequent presence of snow and ice (3B42RT is not updated under such circumstances).

Figure 11 shows the assimilation results for the grids and days with soil moisture observations, using the NLDAS precipitation as a reference. Overall, the method is successful in correcting daily rainfall amount when 3B42RT overestimates precipitation (3B42RT - NLDAS $>0$ ). Mean standard deviation (SD) of $3 B 42 R^{A D J}-$ NLDAS is between 1 and $3 \mathrm{~mm} \mathrm{day}^{-1}$ (statistics provided in Table 3). When 3B42RT underestimates rainfall (3B42RT - NLDAS $<0)$, the assimilation has limited improvement on 3B42RT. This is due to the effect of surface saturation. In terms of adding rainfall, effectiveness of the assimilation is limited under the following two circumstances.

1. The presence of wet conditions or (near) saturation. There is higher probability bringing the surface to saturation (wetter condition) when the assimilation adds rainfall into 3B42RT. However, soil moisture increments are less sensitive to incoming precipitation on wetter soil. Therefore, an error in $\triangle \mathrm{SM}$ often translates into $3 \mathrm{~B} 42 \mathrm{RT} \mathrm{ADJ}_{\mathrm{AD}}$ in a magnified manner.

2. The presence of very heavy precipitation, which typically brings the surface to saturation, hence not results in an update of 3B42RT, is not updated. If, by a small probability, the surface is wet (nearly saturated) but not completely saturated after a heavy rainfall, the updated 3B42RT also suffers from large uncertainty (explained in 1) above).

The effect of the assimilation conditioned on 3B42RT rainfall amount is further evaluated by skill scores. Figure 12 presents probability of detection (POD) and false alarm rate (FAR) in 3B42RT and 3B42RT ${ }_{\mathrm{ADJ}}$, using NLDAS as the reference data set. The rain event threshold is set to be 0.1 and $2 \mathrm{~mm} \mathrm{day}^{-1}$. This is possibly due to lower soil moisture variability in satellite retrievals over the dry, mountainous areas and frequent presence of snow and ice (3B42RT is not updated under such circumstances). For a $0.1 \mathrm{~mm} \mathrm{day}^{-1}$ threshold, both FAR and POD increase in $3 \mathrm{~B}_{4} 2 \mathrm{RT}_{\mathrm{ADJ}} \mathrm{ex}-$ cept for the mountainous region, whereas, for a $2 \mathrm{~mm}_{\text {day }}{ }^{-1}$ threshold, there is only a slight increase in FAR in most of the eastern US region. The overestimation of rain days is also absent when the $2 \mathrm{~mm} \mathrm{day}^{-1}$ event threshold is applied, which suggests that most of the excessive rainy days have a less than $2 \mathrm{~mm} /$ day rain amount. Consistent with Wanders et al. (2015), spatially, larger improvements are found 
Table 2. Error statistics of recovered NLDAS based on $\triangle \mathrm{SM}$ (with added errors) conditioned on first-layer soil wetness for the idealized experiment $\left(\mathrm{mm} \mathrm{day}^{-1}\right)$.

\begin{tabular}{|c|c|c|c|c|c|c|c|c|c|c|}
\hline $\begin{array}{l}\text { [Recovered } \\
\text { NLDAS] - } \\
{[\text { NLDAS] }} \\
\text { mm day }^{-1}\end{array}$ & $\begin{array}{l}\text { [VIC first-layer SM] } \\
-[\text { maximum }]^{*} \\
\mathrm{~mm}\end{array}$ & $<-30$ & -30 to -25 & -25 to -20 & -20 to -15 & -15 to -12 & -12 to -10 & -10 to -9 & -9 to -8 & $>-8$ \\
\hline \multirow[t]{2}{*}{ No error } & Median & 0.04 & 0.03 & 0.02 & 0.02 & 0.02 & 0.03 & 0.03 & 0.04 & 0.16 \\
\hline & IQR & 0.14 & 0.08 & 0.07 & 0.07 & 0.08 & 0.12 & 0.21 & 0.29 & 1.71 \\
\hline \multirow[t]{2}{*}{1.0} & Median & 0.86 & 1.07 & 1.08 & 1.03 & 0.99 & 0.97 & 0.97 & 0.94 & 0.66 \\
\hline & IQR & 1.52 & 1.72 & 1.77 & 1.83 & 1.96 & 2.08 & 2.14 & 2.19 & 2.59 \\
\hline \multirow[t]{2}{*}{2.0} & Median & 0.68 & 1.07 & 1.40 & 1.56 & 1.52 & 1.44 & 1.51 & 1.64 & 1.54 \\
\hline & IQR & 1.76 & 2.09 & 2.88 & 3.45 & 3.63 & 3.73 & 3.73 & 3.73 & 3.91 \\
\hline \multirow[t]{2}{*}{3.0} & Median & 0.15 & 0.80 & 1.20 & 1.41 & 1.47 & 1.51 & 1.65 & 1.84 & 1.88 \\
\hline & IQR & 1.36 & 2.16 & 3.04 & 3.73 & 3.74 & 3.79 & 4.34 & 5.24 & 5.47 \\
\hline \multirow[t]{2}{*}{4.0} & Median & 0.22 & 0.56 & 0.83 & 1.15 & 1.30 & 1.40 & 1.63 & 1.88 & 1.97 \\
\hline & $\mathrm{IQR}$ & 0.99 & 2.36 & 2.48 & 3.99 & 4.05 & 4.70 & 5.53 & 5.52 & 5.63 \\
\hline \multirow[t]{2}{*}{5.0} & Median & 0.00 & 0.15 & 0.52 & 0.90 & 1.10 & 1.27 & 1.54 & 1.81 & 1.89 \\
\hline & IQR & 1.62 & 2.54 & 2.91 & 4.43 & 4.51 & 5.95 & 5.90 & 5.79 & 7.04 \\
\hline
\end{tabular}

* First-layer soil depth is $100 \mathrm{~mm}$ with a SM capacity of $\sim 45 \mathrm{~mm}$, depending on porosity.

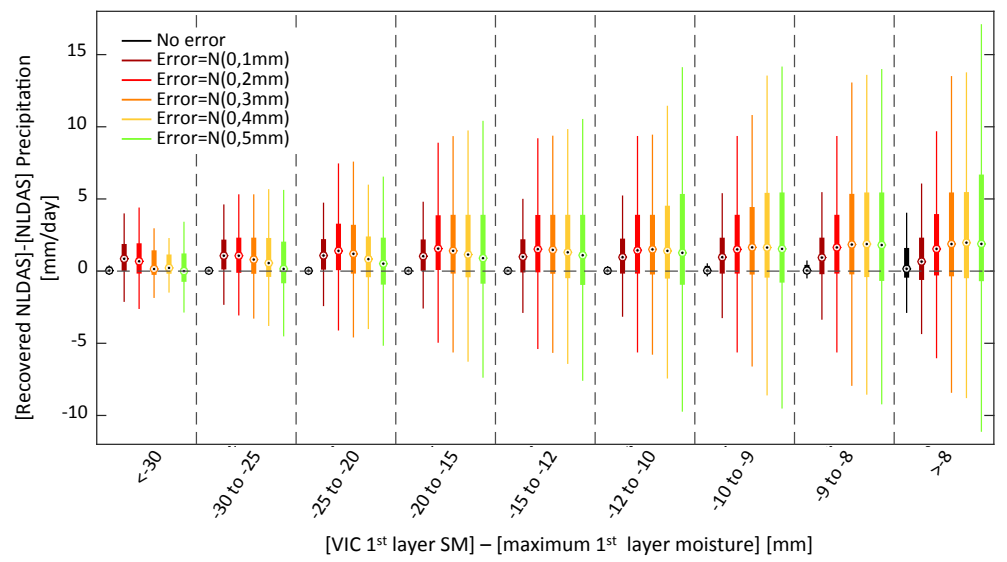

Figure 7. Error in recovered NLDAS precipitation given surface moisture condition. Recovered NLDAS is based on using "truth" soil moisture and soil moisture with normal error: $N(0.1 \mathrm{~mm}), N(0.2 \mathrm{~mm}), N(0.3 \mathrm{~mm}), N(0.4 \mathrm{~mm})$ and $N(0.5 \mathrm{~mm})$. Statistics provided in Table 2 .

in the central US. The area coincides where higher AMSRE/LSMEM $\triangle \mathrm{SM}$ accuracy is found (non-mountainous regions with little urbanization and light vegetation). Despite the regional variability, these excessive rainy days are a result of the high-frequency noise in AMSR-E/LSMEM soil moisture retrievals identified by Pan et al. (2004) and Wanders et al. (2015).

The applied method is ineffective for light rainfall $<\mathrm{mm}$, where the adjustment tends to over-correct precipitation by adding excessive rainfall - mostly the result of the high-frequency AMSR-E noise. The MAE of light rainfall $\left(<2 \mathrm{~mm} \mathrm{day}^{-1}\right)$ increased from $0.65 \mathrm{~mm} \mathrm{day}^{-1}$ in 3B42RT to $0.99 \mathrm{~mm} \mathrm{day}^{-1}$ in $3 \mathrm{~B} 42 \mathrm{RT}_{\mathrm{ADJ}}$. On the other hand, satellite soil moisture assimilation is very effective in correcting satellite precipitation higher than $2 \mathrm{~mm}$ day $^{-1}$ : the MAE of medium to large rainfall $\left(\geq 2 \mathrm{~mm} \mathrm{day}^{-1}\right)$ decreased from
$7.07 \mathrm{~mm} \mathrm{day}^{-1}$ in 3B42RT to $6.55 \mathrm{~mm} \mathrm{day}^{-1}$ in 3B42RT $\mathrm{ADJ}$. The effect of the assimilation is different over the western mountainous region, the north-to-south central US band and the eastern US.

The western mountainous region has a dry climatology with more frequent rainfall in small amounts. The white noise in $\triangle \mathrm{SM}$, negatively impacting $3 \mathrm{~B} 42 \mathrm{RT}_{\mathrm{ADJ}}$, is comparable to the positive improvement brought by actual light rainfall signals in $\triangle \mathrm{SM}$. Therefore, the assimilation of $\Delta \mathrm{SM}$ has no significant impact in these regions.

The north-to-south band over the central US experiences more medium to high $\left(\geq 2 \mathrm{~mm} \mathrm{day}^{-1}\right)$ rainfall. In addition, the region is lightly vegetated (annual mean LAI $<1$ ) with low elevation $(<1500 \mathrm{~m})$, where soil moisture retrievals are of higher accuracy. Soil moisture climatology is wetter in the west, causing larger variations in $3 \mathrm{~B}_{42} \mathrm{RT}_{\mathrm{ADJ}}$ error from the 
a) $\triangle \mathrm{SM}$

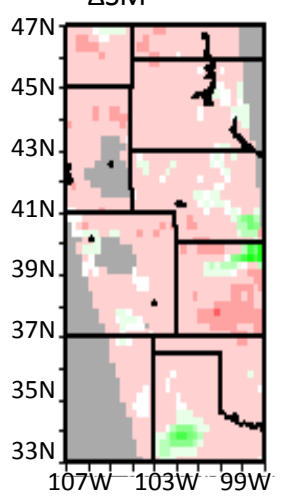

b) $3 \mathrm{~B} 42 \mathrm{RT}$

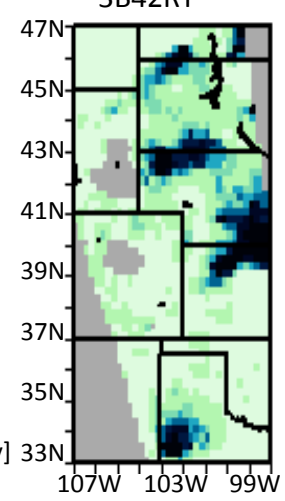

c)

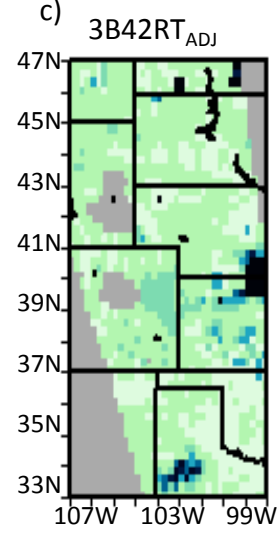

d) NLDAS

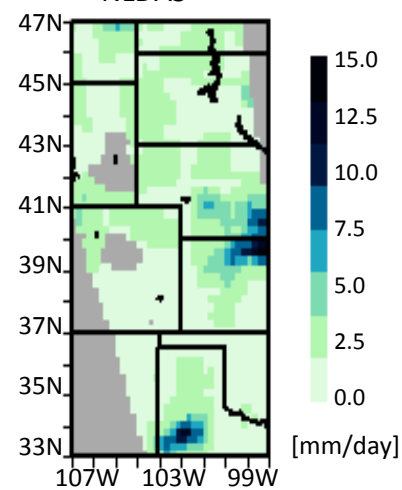

Figure 8. 26 May 2006 rainfall pattern in 3B42RT (b) against NLDAS (d) as detected by AMSR-E/LSMEM $\triangle$ SM (a), and recovered rainfall field (3B42RT $\left.\mathrm{ADJ}_{\mathrm{A}}\right)$ by assimilating AMSR-E/LSMEM $\triangle \mathrm{SM}$ (c). Gray shading shows area without soil moisture retrievals.

a) $[$ NLDAS] \& [AMSR-E/LSMEM $\triangle S M]$

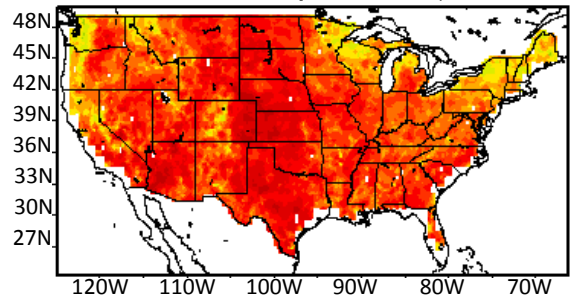

b) $[3 B 42 R T] \&[A M S R-E / L S M E M \triangle S M]$

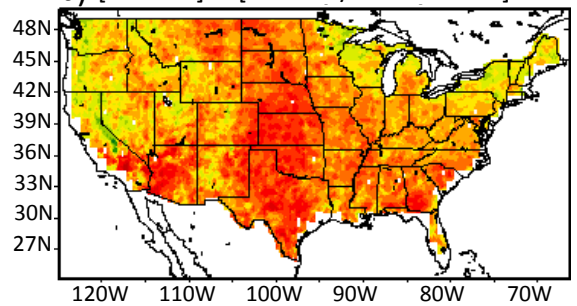

C) $\left[3 B 42 R_{A D}\right] \&[A M S R-E / L S M E M \triangle S M]$

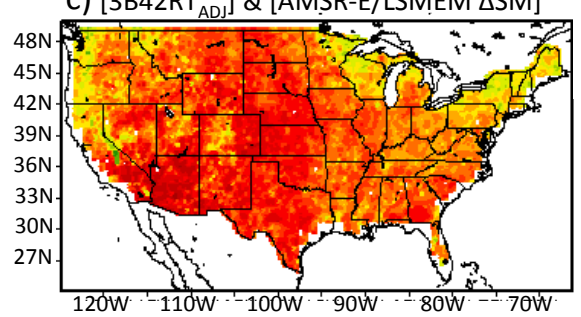

d)

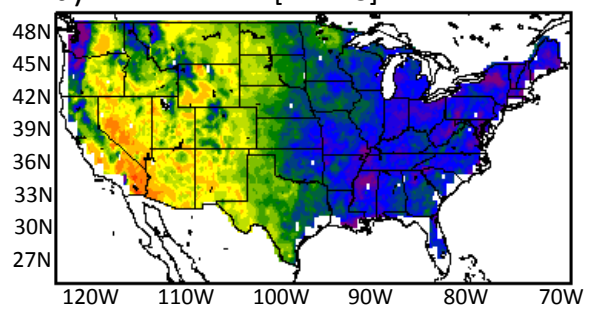

e)

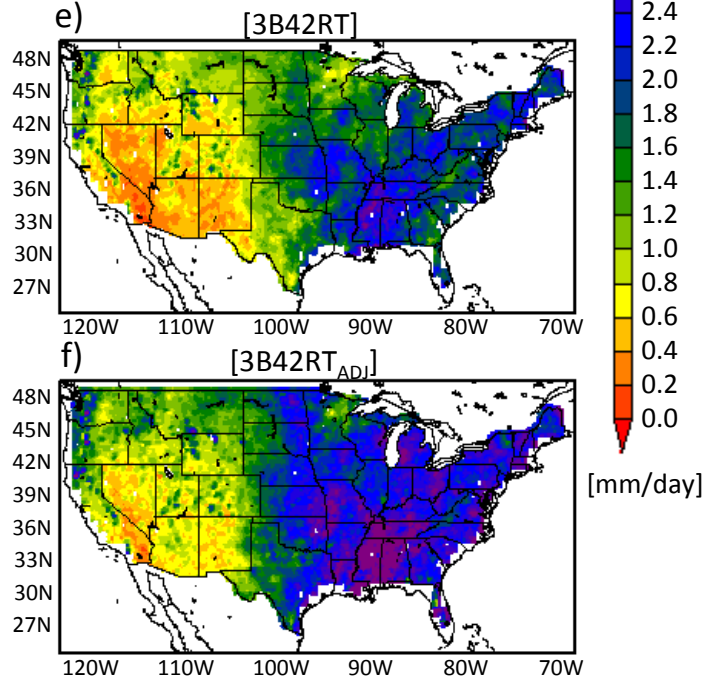

Figure 9. Pearson correlation coefficient between AMSR-E/LSMEM $\triangle$ SM and precipitation (from 1 January 2003 to 31 July 2007): (a) NLDAS, (b) 3B42RT and (c) 3B42RT $\mathrm{ADJ}$; annual mean precipitation in (d) NLDAS, (e) 3B42RT and (f) $3 \mathrm{~B}_{42} \mathrm{RT}_{\mathrm{ADJ}}$ of time steps with AMSR-E/LSMEM $\triangle$ SM retrievals.

white noise $\Delta \mathrm{SM}$ (as discussed in Sect. 3.2). Despite that, satellite soil moisture is most effective correcting medium to large rainfall under normal surface conditions.

The decreased skill in $3 \mathrm{~B} 42 \mathrm{RT}_{\mathrm{ADJ}}$ over the eastern US is primarily attributed to both precipitation and soil moisture climatology, a wet climate with more medium to high rainfall, neither of which is suitable for soil moisture assimilation.
In summary, the high-frequency noise in soil moisture product causes a major limitation. The noise impacts adjusted precipitation by introducing false alarm rain days. It is difficult to distinguish the noise and retrieve the true rainfall signals. A remedy to prevent the excessive rain days is applying a cutoff $\Delta \mathrm{SM}$ threshold when rain days are added, at the expense of neglecting a part of the true rainfall signals. Figure 13 shows the probability of added rainy 

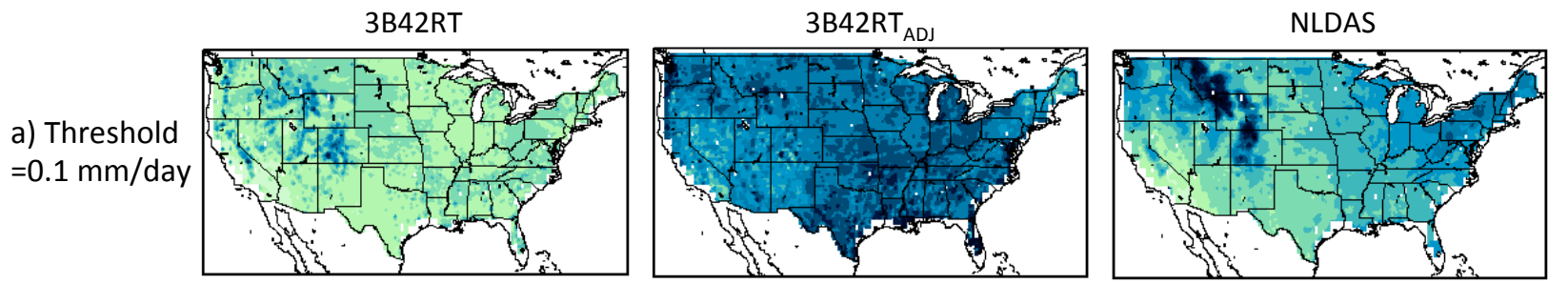

1.0

0.8

0.6
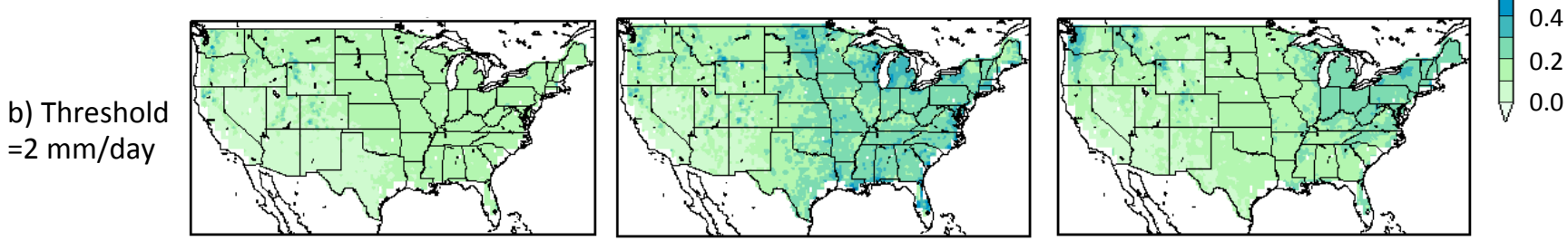

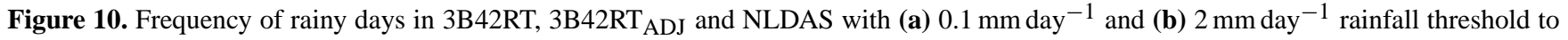
define a rain day.

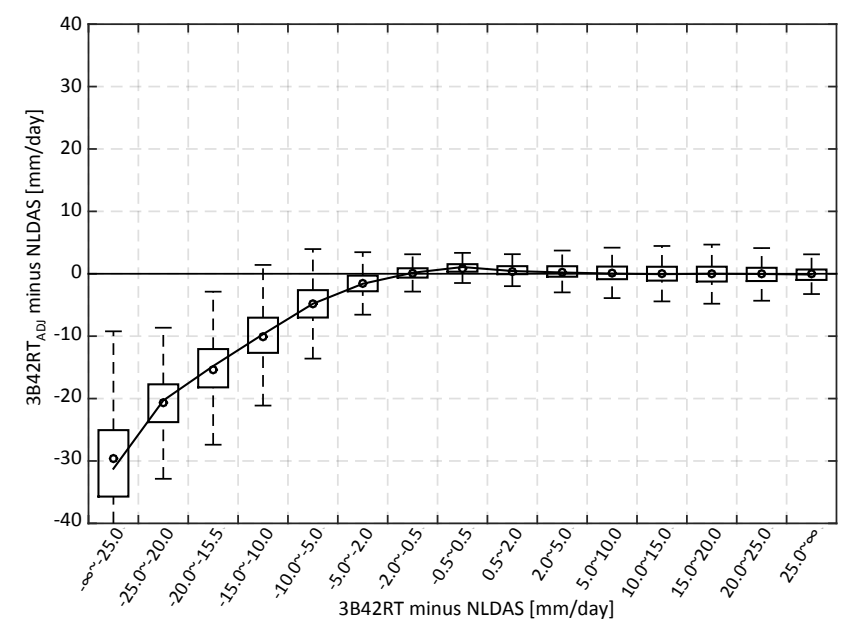

Figure 11. Distribution of $3 B 42 R T$ and $3 B 42 R^{A D J}$ precipitation error compared to NLDAS. Statistics are provided in Table 3.

days being consistent with NLDAS (NLDAS $>0 \mathrm{~mm}$ day $^{-1}$ ) with respect to $\triangle \mathrm{SM}$. When a new rainy day is added (3B42RT $=0 \mathrm{~mm} \mathrm{day}^{-1}, 3 \mathrm{~B} 42 \mathrm{RT}_{\mathrm{ADJ}}>0 \mathrm{~mm} \mathrm{day}^{-1}$ ) based on AMSR-E/LSMEM $\Delta$ SM of $2 \mathrm{~mm}_{\text {day }}{ }^{-1}$, there's approximately $78 \%$ chance that the added rain day is a true event (NLDAS $>0 \mathrm{~mm} \mathrm{day}^{-1}$ ); That is, $\sim 22 \%$ chance that it is a false alarm (NLDAS $=0 \mathrm{~mm} \mathrm{day}^{-1}$ ). When AMSRE/LSMEM $\triangle \mathrm{SM}$ is larger than $2 \mathrm{~mm} \mathrm{day}^{-1}$, the probability of added rainy days being true event is even higher, up to $90 \%$ chance. Here we applied a threshold of $2 \mathrm{~mm} \mathrm{day}^{-1}$ to AMSR-E/LSMEM $\triangle \mathrm{SM}$. That is, when new rainy days are introduced (3B42RT $>0,3 B 42 R T A D J>0)$, we discard the update and keep the no-rain day if AMSR-E/LSMEM soil moisture increment is below $2 \mathrm{~mm}$. Note that, the probability of the false alarms depends on soil moisture climatology: the wetter soil moisture climatology, the larger uncertainty in the signal. Therefore, this threshold should vary in accordance with local soil moisture climatology, i.e., a larger threshold over the wetter eastern US and a smaller threshold over the drier western US. Nevertheless, after the $2 \mathrm{~mm} \mathrm{day}^{-1} \Delta \mathrm{SM}$ threshold is applied, expectedly, the statistics are largely improved: FAR is decreased significantly from 0.519 (wo. $\triangle \mathrm{SM}$ threshold) to 0.066 (w. $\triangle \mathrm{SM}$ threshold). MAE of light rainfall $\left(<2 \mathrm{~mm} \mathrm{day}^{-1}\right)$ in 3B42RT $\mathrm{ADJ}_{\mathrm{AJ}}$ decreased from 0.99 to $0.64 \mathrm{~mm} \mathrm{day}^{-1}$, compared to $0.65 \mathrm{~mm} \mathrm{day}^{-1}$ in 3B42RT. For medium to large 3B42RT rainfall $\left(\geq 2 \mathrm{~mm} \mathrm{day}^{-1}\right)$, it effectively increased POD (0.362 in $3 \mathrm{~B} 42 \mathrm{RT}$ vs. 0.386 in $3 \mathrm{~B}_{42} \mathrm{RT}_{\mathrm{ADJ}}$ w. $\Delta \mathrm{SM}$ threshold) and decreased FAR (0.037 in 3B42RT vs. 0.030 in $3 \mathrm{~B} 42 \mathrm{RT}_{\mathrm{ADJ}}$ w. $\triangle \mathrm{SM}$ threshold). Further work is needed to characterize, distinguish and decrease the high-frequency noise in SM retrievals. Figure 13 gives an example of evaluating the impact of SM uncertainties in assimilation as curves derived over different topography can be quantitatively compared.

\section{Comparison to other studies}

Many other studies have utilized satellite microwave brightness temperatures or soil moisture retrievals to constrain satellite precipitation estimates (Pellarin et al., 2008), estimate precipitation (e.g., Brocca et al., 2013) or improve precipitation estimates through assimilation (Crow et al., 2009, 2011). Here, we review their approaches and findings in light of the results of this study, and compare our results with some of these studies to gain insight into their robustness and consistency.

Pellarin et al. (2008) used the temporal variations of the AMSR-E $6.7 \mathrm{GHz}$ brightness temperature (TB) normalized polarization difference, $\mathrm{PR}=\left(\mathrm{TB}_{\mathrm{V}}-\mathrm{TB}_{\mathrm{H}}\right) /\left(\mathrm{TB}_{\mathrm{V}}+\mathrm{TB}_{\mathrm{H}}\right)$, to screen out anomalous precipitation events from a 4day cumulative satellite-estimated precipitation (EPSAT-SG: Chopin et al., 2005) from 22 to 26 June 2004 over a 
a) Threshold=0.1mm/day
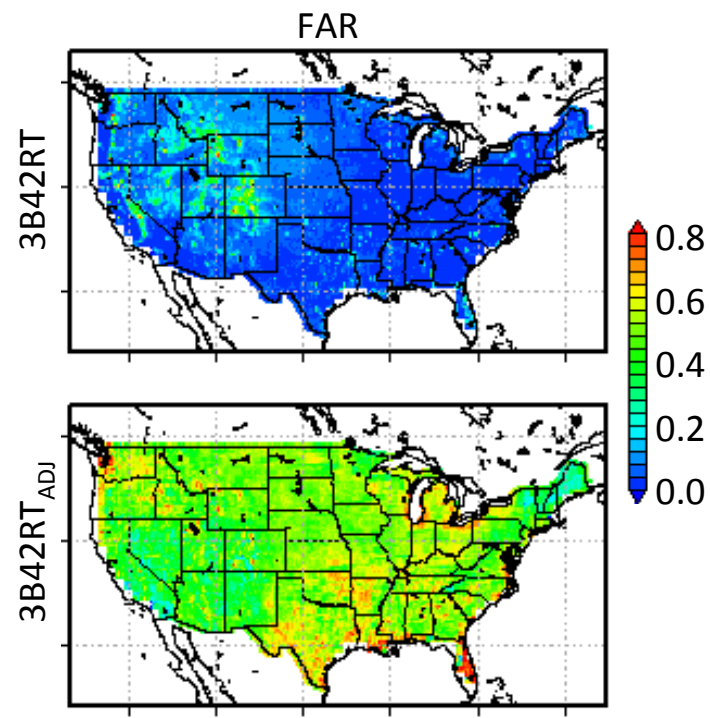

b) Threshold=2mm/day
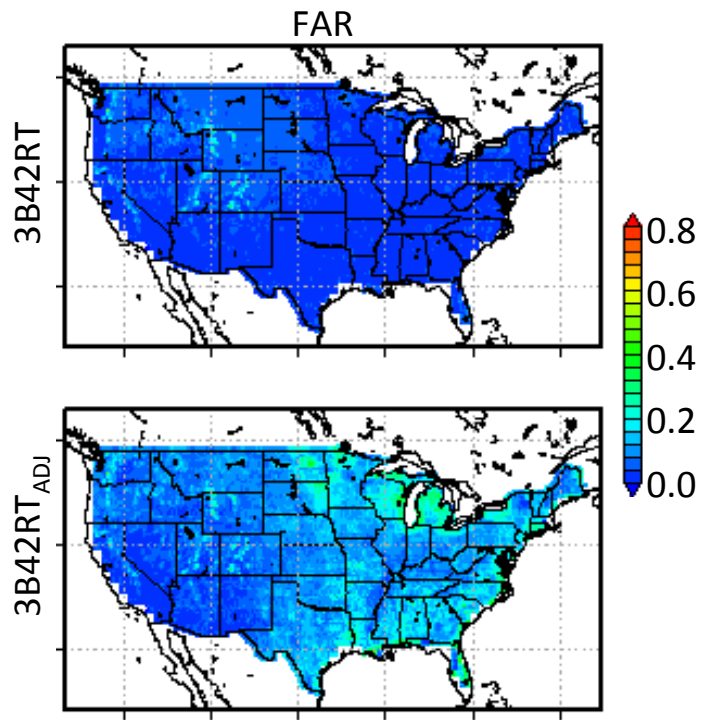
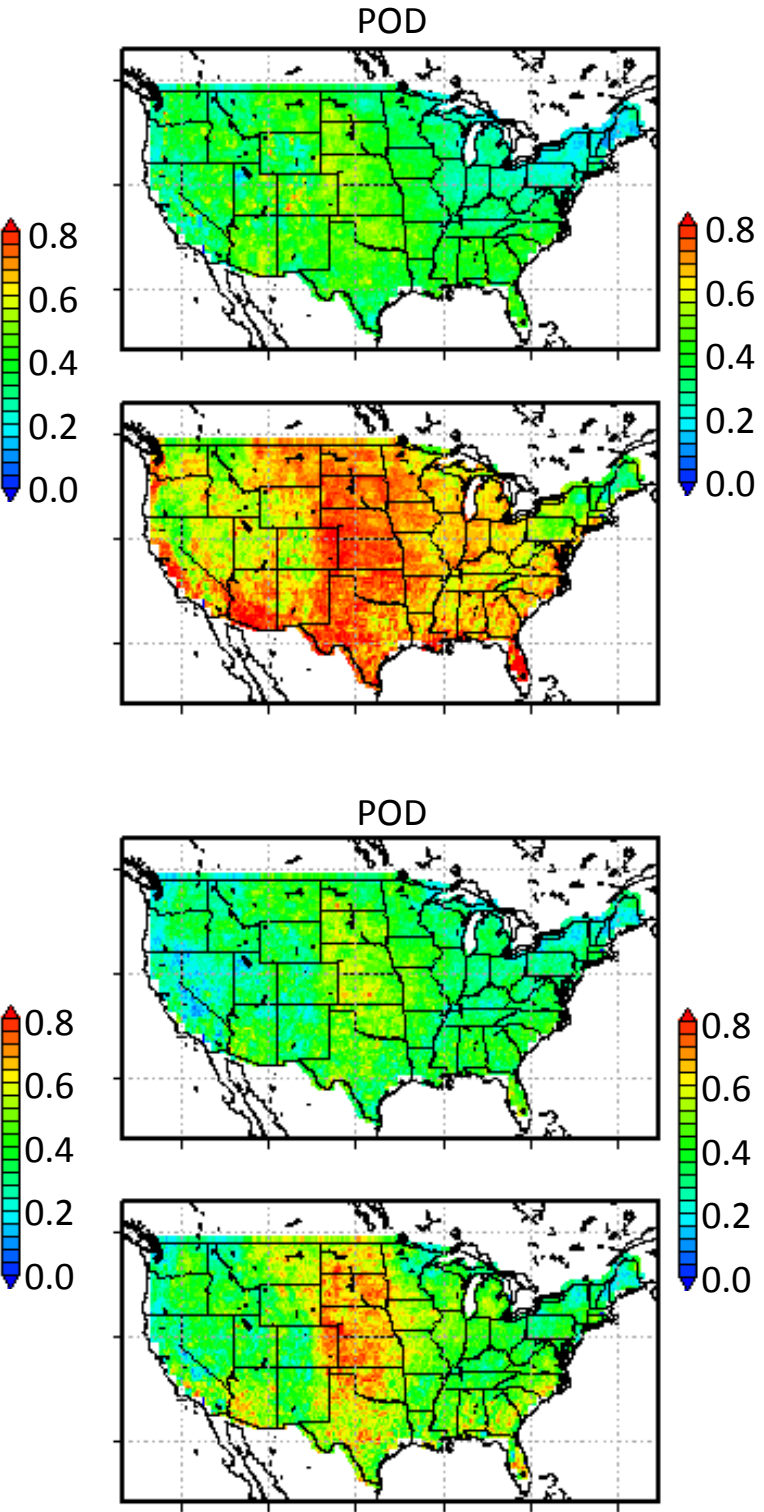

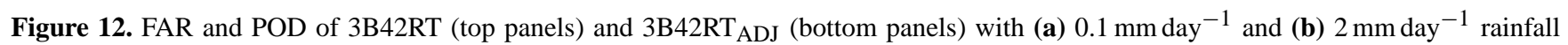
thresholds to define a rain event.

$100 \times 125 \mathrm{~km}$ box centered over Niger in West Africa. This was extended in Pellarin et al. (2013), where an API-based water balance model was used to correct three different satellite precipitation products (CMORPH, TRMM-3B42 and PERSIANN) over a 4-year period in West Africa at three $0.25^{\circ}$ grids in Niger, Benin and Mali). The new algorithm was evaluated by comparing the corrected precipitation to estimates over the $0.25^{\circ}$ grids from ground-based precipitation measurements. A sequential assimilation approach was applied where AMSR-E C-band TB measurements were used to estimate a simple multiplicative factor to the precipitation estimates in order to minimize the difference between ob- served (AMSR-E) and simulated TBs in terms of root mean square error (RMSE). The results show improvements over those found in Pellarin et al. (2009). Specifically, the Pellarin et al. (2013) study shows that the proposed methodology produces an improvement of the RMSE at daily, decadal and monthly timescales and at the three locations. For instance, the RMS mean error decreases from 7.7 to $3.5 \mathrm{~mm} \mathrm{day}^{-1}$ at the daily timescale in Niger and from 18.3 to $7.7 \mathrm{~mm} \mathrm{day}^{-1}$ at the decadal timescale in Mali.

Crow et al. $(2003,2009,2011)$ demonstrated the effectiveness of the assimilation of remotely sensed microwave brightness temperatures or retrieved soil moisture in esti- 


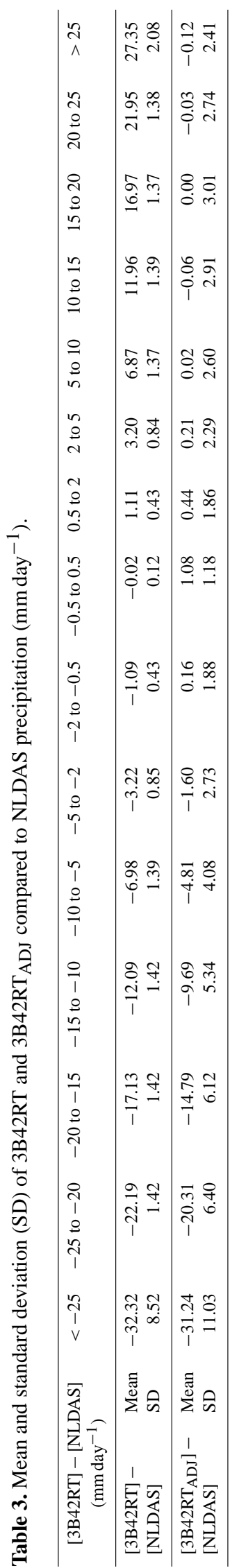

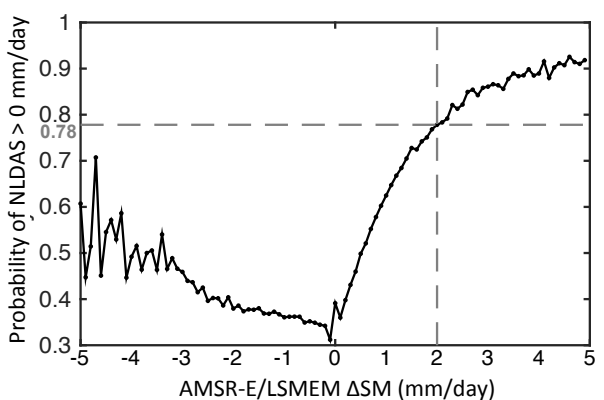

Figure 13. Probability that the added rainy days $\left(3 \mathrm{~B} 42 \mathrm{RT}=0 \mathrm{~mm} \mathrm{day}^{-1}, \quad 3 \mathrm{~B} 42 \mathrm{RT}_{\mathrm{ADJ}}>0 \mathrm{~mm} \mathrm{day}^{-1}\right)$ are true rain events (NLDAS $>0 \mathrm{~mm}^{-1}{ }^{-1}$ ) given corresponding AMSRE/LSMEM $\Delta$ SM.

mating precipitation based on airborne measurements over the Southern Great Plains (USA) region (Crow et al., 2003); 2- to 10-day accumulated precipitation within a simple API water budget model and assimilation scheme over CONUS (Crow et al., 2009); and 3-day, $1^{\circ}$ precipitation accumulation over three African Monsoon Multidisciplinary Analysis (AMMA) sites in West Africa with an enhanced assimilation scheme and an API moisture model (Crow et al., 2011). Crow et al. (2009) recommend against estimating precipitation at a larger scale than 3 days based on assimilating AMSR-E/LSMEM soil moisture.

Brocca et al. (2013) estimated precipitation by inverting the water budget equation such that precipitation could be estimated from changes in soil moisture. The inverted equation was calibrated using in situ, 4-day averaged observations at two sites in Spain and Italy. In Brocca et al. (2014), the same approach was used globally to estimate daily precipitation at $1^{\circ}$ spatially. Five-day cumulated rainfall estimates are derived from three satellite-derived soil moisture data sets (AMSR-E LPRM, ASCAT and SMOS), and linearly interpolated to daily values, for their precipitation estimation algorithm. No formal data assimilation was carried out. The newly created precipitation data set was compared to two satellite precipitation products (TRMM3B42RT, GPCC) and two gauge-based precipitation products (GPCP, ERA-Interim). Five-day accumulated rainfall data, aggregated to a $1^{\circ}$ spatial resolution, are considered in their assessment analyses with promising results. But, they do note that their approach has "poor scores in reproducing daily rainfall data". Ciabatta et al. (2015) derived daily rainfall product using ASCAT over Italy and integrated it with TMPA 3B42RT precipitation. The merged product also shows promising results.

In the study reported here, four advances have been made over these earlier studies: (i) we adopted a state-of-the-art dynamic land surface model that has demonstrated high skill in simulating soil moisture when driven by high-quality precipitation data (Schaake et al., 2004); (ii) we applied a state-ofthe-art data assimilation procedure based on particle filtering 
so as to extract (and hopefully maximize) the information content from the satellite most effectively; (iii) we increased the resolution of the precipitation estimation window down to 1 day, exceeding the conclusions in these earlier studies that the finest temporal resolution is 3 to 5 days. Additionally we increased (or matched) the spatial resolution to $0.25^{\circ}$, limited primarily by the satellite soil moisture product resolution; and (iv) previous studies are based on the assumption that the SM retrievals are $100 \%$ accurate and contain no errors. We evaluated this assumption by analyzing the impact of uncertainties associated with the soil moisture retrievals. These advances offer important benefits when satellite precipitation products are used for applications such as flood forecasting. Admittedly, by aggregating in space and time, the improvement is more robust since some errors are averaged out.

Wanders et al. (2015) performed a comprehensive intercomparison study using multiple satellite soil moisture and land surface temperature (LST) data at fine temporal scale (3-hourly). Compared to their study, ours focuses on using soil moisture exclusively from one satellite and retrieval algorithm, and in improvements to the assimilation algorithm, specifically, (i) the longer temporal period (2010-2011 in Wanders et al. (2015) vs. 2002-2007 in this study), (ii) the temporal resolution (3-hourly vs. daily), and (iii) the particle generation and bias-correction method. We present in the paper improvements in the generation of rain particles and the bias-correction of the satellite soil moisture observations, as well as enhancements to the assimilation algorithm to maximize the information that can be gained from using soil moisture alone in adjusting precipitation. Due to the very strong and complicated spatial structure of precipitation, that is non-Gaussian and non-stationary in both time and space, a more advanced method is applied to generate possible precipitation fields than used or presented in earlier studies or in Wanders et al. (2015). Furthermore, a more advanced biascorrection method is also applied to account for the reported problems (Wanders et al., 2015) in the second-order statistics of the soil moisture retrievals, and (iv) SM retrieval products (and overpasses) used in assimilation. Our improved results are based on soil moisture retrievals from ascending overpasses only (vs. both descending and ascending overpasses from multiple data sets, i.e., AMSR-E/LSMEM, ASCAT and SMOS). Our exclusive focus on the usefulness of soil moisture product promises more applicability especially for improving satellite precipitation from the Global Precipitation Mission products. The descending overpasses have generally better performance than the ascending, suggesting the potentials of further improvements.

A quantitative comparison of Wanders et al. (2015) and our results is provided below. Despite the different time periods between Wanders et al. (2015, 2010-2011) and in our study (2002-2007), Wanders et al. (2015) show decreasing POD $(-15.0$ to $-46.4 \%$, depending on the different products used) and FAR ( -47.2 to $-89.1 \%$, depending on the different products used) for all rainfall after assimilation using either (single or multiple) SM products alone or SM + LST data combined (see Table 4 of Wanders et al., 2015). While in our study, after applying $\Delta$ SM threshold, medium to large $3 \mathrm{~B} 42 \mathrm{RT}$ ADJ rainfall $\left(\geq 2 \mathrm{~mm} \mathrm{day}^{-1}\right)$ has an increase in POD $(+6.6 \%)$ and decrease in FAR $(-18.9 \%)$. Furthermore, the significant dry bias in adjusted precipitation (see Fig. 6 of Wanders et al., 2015) is not present in our results (Fig. 9). This is due to improvements in our precipitation ensemble generation and bias correction scheme. Wanders et al. (2015) applied an additional step generating precipitation particles sampling from a $3 \times 3$ window that overeliminates most of the excessive rainfall along with some real signal. We suggest loosening this constraint to a larger window size or to sample from adjusted precipitation instead of original 3B42RT precipitation. However, sampling from adjusted precipitation at each time step would significantly increase the computational demand, limiting the potential for a global application at high temporal/spatial resolution.

Furthermore, the outcome is quite different for the distribution of soil moisture retrievals after pre-processing (Fig. 9 of Wanders et al. (2015) vs. Fig. 4 in our study) due to the different methods used. After pre-processing, distributions of soil moisture retrievals are more similar to that of NLDAS precipitation forced, VIC modeled first-layer soil moisture. CDF-matching used by Wanders et al. (2015) is based on the assumption that satellite soil moisture and modeled soil moisture respond to heavy rainfall in the same way - essentially having a rank correlation of 1 . However, that is not observed because of the shallower detection depth of the satellite soil moisture. On the other hand, using the pre-processing method presented in this study, the signal of near-saturation in AMSR-E/LSMEM $\triangle \mathrm{SM}$ tends to be overestimated after pre-processing, which indicates a heavy rain event that is often accompanied with surface saturation and thus does not provide effective information for the assimilation. The other benefit of the second-order polynomial regression lies in its non-linearity. An error in the soil moisture product impacts the precipitation adjustment in a predictable way, allowing for a more systematic post-processing treatment. Based on the known error characteristics, we demonstrate a potential remedy to deal with the error by applying a $2 \mathrm{~mm}_{\text {day }}{ }^{-1}$ cutoff $\Delta \mathrm{SM}$ threshold. Meanwhile, it is also highlighted that the cutoff threshold should be variable and positively correlated with local soil moisture climatology. We acknowledge that the soil moisture product used in Wanders et al. (2015) is a blended product of multiple satellite soil moisture data sets. It is not clear how its error characteristics impact the adjusted precipitation. 


\section{Conclusion and discussion}

Based on the retrieved soil moisture from AMSR-E using the LSMEM retrieval algorithm, we propose an assimilation procedure to integrate soil moisture information into the VIC land surface model so as to improve real-time, satellite precipitation estimates. The ability to estimate rainfall amount is now enhanced with the above improvements, especially for correcting medium rainfall amounts. However, constrained by the noise in AMSR-E TBs and thus soil moisture retrievals, the assimilation is not effective in detecting missed rainfall events. The improved precipitation estimates, referred to as $3 \mathrm{~B} 42 \mathrm{RT}_{\mathrm{ADJ}}$ estimates, are overall consistent in reproducing the spatial pattern and time series of daily rainfall from NLDAS precipitation. The results illustrate the potential benefits of using data assimilation to merge satellite retrievals of surface soil moisture into a land surface model forced with real-time precipitation. Potentially the method can be applied globally for areas meeting vegetation cover and surface condition constraints that allows for soil moisture retrievals. Under these conditions, the approach can provide a supplementary source of information for enhancing the quality of satellite rainfall estimation, especially over poorly gauged areas like Africa.

Nonetheless, some caution is required. The results of this study show that the adjusted real-time precipitation tends to add additional rain (frequency) resulting in more time steps with rain but lower regional average in the western US and slightly higher regional average in the eastern US. It is also noticed that the precipitation adjustments are insensitive under saturated soil moisture conditions. A wetter surface magnifies any error associated with satellite observation by incorrectly adjusting precipitation. These errors, mixed with the "real" signal, generally add approximately $\sim 2 \mathrm{~mm}$ of precipitation (or higher), depending on the soil moisture climatology. It is important to consider these circumstances when observations are used so as to avoid introducing additional error. With these identified limitations, continued research is needed to assess the biases in the real-time precipitation retrievals on a local to regional basis so the assimilation system can be modified accordingly.

The assimilation scheme used here assumed that the errors were attributed to the real-time precipitation retrievals, but the precipitation estimates after adjustment includes errors from additional sources. The two primary sources are errors in soil moisture retrievals and errors in the land surface model that include model parameterizations (poorly or insufficiently represented processes as well as scale issues) and parameter errors (insufficient calibration). There are also errors in other model forcing fields besides precipitation. Further studies are needed to assess the attribution of these error sources to the total error. Such research will further improve the use of real-time satellite-based precipitation for global flood monitoring.
Besides the clear, heavy dependency of the assimilation effectiveness on the accuracy of satellite soil moisture product, it is also important to acquire adequate knowledge on the error characteristics of satellite soil moisture retrievals. Knowledge of the soil moisture errors could be important and the assimilation methods (including precipitation ensemble generation and pre-/post-processing method) should be chosen accordingly. On the other hand, the presence of data gaps between overpasses could be a large source of uncertainty with data assimilation. Further effort towards reliable spatialtemporal continuous (gap-filled) satellite soil moisture data sets is needed.

While it has been illustrated in this study that the enhancement of real-time satellite precipitation estimates can be realized through an assimilation approach using satellite soil moisture data products and a particle filter, additional satellite-based observations (e.g., multi-sensor soil moisture products) or variables (e.g., land surface temperatures as shown in Wanders et al. (2015), inundated areas) could be added/replaced in the assimilation process with different levels of complexity, e.g., by applying constraints on the particle generation. This opens up a great number of opportunities in using space-borne observations for supplementing direct retrievals of precipitation.

Acknowledgements. This research was supported through NASA grant NNX13AG97G (Multi-sensor enhancement of real-time satellite precipitation retrievals for improved drought monitoring) under the Precipitation Measurement Mission. Part of this research was financially supported by NWO Rubicon 825.15.003. This support is gratefully acknowledged.

Edited by: W. Wagner

\section{References}

Brocca, L., Melone, F., Moramarco, T., and Morbidelli, R.: Antecedent wetness conditions based on ERS scatterometer data, $\mathbf{J}$ Hydrol., 364, 73-87, doi:10.1016/j.jhydrol.2008.10.007, 2009.

Brocca, L., Moramarco, T., Melone, F., and Wagner, W.: A new method for rainfall estimation through soil moisture observations, Geophys. Res. Lett., 40, 853-858, doi:10.1002/grl.50173, 2013.

Brocca, L., Ciabatta, L., Massari, C., Moramarco, T., Hahn, S., Hasenauer, S., Kidd, R., Dorigo, W., Wagner, W., and Levizzani, V.: Soil as a natural rain gauge: Estimating global rainfall from satellite soil moisture data, J. Geophys. Res.-Atmos., 119, 51285141, doi:10.1002/2014JD021489, 2014.

Chopin, F., Berges, J., Desbois, M., Jobard, I., and Lebel, T.: Satellite Rainfall Probability and Estimation. Application to the West Africa During the 2004 Rainy Season, AGU Spring Meet. Abstr. A12, New Orleans, Louisiana, USA, 2005.

Ciabatta, L., Brocca, L., Massari, C., Moramarco, T., Puca, S., Rinollo, A., Gabellani, S., and Wagner, W.: Integration of Satellite Soil Moisture and Rainfall Observations over the Italian Ter- 
ritory, J. Hydrometeorol., 16, 1341-1355, doi:10.1175/JHM-D14-0108.1, 2015.

Crow, W. T.: Correcting land surface model predictions for the impact of temporally sparse rainfall rate measurements using an ensemble Kalman filter and surface brightness temperature observations, J. Hydrometeorol., 4, 960-973, 2003.

Crow, W. T., Huffman, G. J., Bindlish, R., and Jackson, T. J.: Improving Satellite-Based Rainfall Accumulation Estimates Using Spaceborne Surface Soil Moisture Retrievals, J. Hydrometeorol., 10, 199-212, doi:10.1175/2008JHM986.1, 2009.

Crow, W. T., Van Den Berg, M. J., Huffman, G. J., and Pellarin, T.: Correcting rainfall using satellite-based surface soil moisture retrievals: The Soil Moisture Analysis Rainfall Tool (SMART), Water Resour. Res., 47, 1-15, doi:10.1029/2011WR010576, 2011

Dee, D. P., Uppala, S. M., Simmons, A. J., Berrisford, P., Poli, P., Kobayashi, S., Andrae, U., Balmaseda, M. A., Balsamo, G., Bauer, P., Bechtold, P., Beljaars, A. C. M., Van de Berg, L., Bidlot, J., Bormann, N., Delsol, C., Dragani, R., Fuentes, M., Geer, A. J., Haimberger, L., Healy, S. B., Hersbach, H., Hólm, E. V., Isaksen, L., Kållberg, P., Köhler, M., Matricardi, M., McNally, A. P., Monge-Sanz, B. M., Morcrette, J. J., Park, B. K., Peubey, C., De Rosnay, P., Tavolato, C., Thépaut, J. N., and Vitart, F.: The ERA-Interim reanalysis: Configuration and performance of the data assimilation system, Q. J. Roy. Meteorol. Soc., 137, 553597,2011

Ebert, E. E., Janowiak, J. E., and Kidd, C.: Comparison of near-real-time precipitation estimates from satellite observations and numerical models, B. Am. Meteorol. Soc., 88, 47-64, doi:10.1175/BAMS-88-1-47, 2007.

Ek, M. B., Xia, Y., Wood, E., Sheffield, J., Luo, L., Lettenmaier, D., Livneh, B., Mocko, D., Cosgrove, B., Meng, J., Wei, H., Koren, V., Schaake, J., Mo, K., Fan, Y., and Duan, Q.: North American Land Data Assimilation System Phase 2 (NLDAS-2): Development and Applications, GEWEX Newsl., 21, 5-7, 2011.

Francois, C., Quesney, A., and Ottlé, C.: SAR Data into a Coupled Land Surface-Hydrological Model Using an Extended Kalman Filter, J. Hydrometeorol., 4, 473-487, doi:10.1175/15257541(2003)4<473:SAOESD>2.0.CO;2, 2003.

Huffman, G. J., Bolvin, D. T., Nelkin, E. J., Wolff, D. B., Adler, R. F., Gu, G., Hong, Y., Bowman, K. P., and Stocker, E. F.: The TRMM Multisatellite Precipitation Analysis (TMPA): Quasi-Global, Multiyear, Combined-Sensor Precipitation Estimates at Fine Scales, J. Hydrometeorol., 8, 38-55, doi:10.1175/JHM560.1, 2007.

Huffman, G. J., Adler, R. F., Bolvin, D. T., and Nelkin, E. J.: The TRMM Multi-satellite Precipitation Analysis (TMPA), in: Satellite Rainfall Applications for Surface Hydrology, Springer Netherlands, 3-22, 2010.

Kalman, R. E.: A New Approach to Linear Filtering and Prediction Problems, J. Basic Eng., 82, 35-45, doi:10.1115/1.3662552, 1960.

Kerr, Y. H., Waldteufel, P., Richaume, P., Wigneron, J.-P., Ferrazzoli, P., Mahmoodi, A., Al Bitar, A., Cabot, F., Gruhier, C., Juglea, S. E., Leroux, D., Mialon, A., and Delwart, S.: The SMOS Soil Moisture Retrieval Algorithm, IEEE T. Geosci. Remote, 50, 1384-1403, doi:10.1109/TGRS.2012.2184548, 2012.

Liang, X., Lettenmaier, D. P., Wood, E. F., and Burges, S. J.: A simple hydrologically based model of land surface water and en- ergy fluxes for general circulation models, J. Geophys. Res., 99 14415-14428, doi:10.1029/94JD00483, 1994.

Liang, X., Wood, E. F., and Lettenmaier, D. P.: Surface soil moisture parameterization of the VIC-2L model: Evaluation and modification, Global Planet. Change, 13, 195-206, doi:10.1016/09218181(95)00046-1, 1996.

Massari, C., Brocca, L., Moramarco, T., Tramblay, Y., and Didon Lescot, J.-F.: Potential of soil moisture observations in flood modelling: estimating initial conditions and correcting rainfall, Adv. Water Resour., 74, 44-53, doi:10.1016/j.advwatres.2014.08.004, 2014.

Matgen, P., Fenicia, F., Heitz, S., Plaza, D., de Keyser, R., Pauwels, V. R. N., Wagner, W., and Savenije, H.: Can ASCAT-derived soil wetness indices reduce predictive uncertainty in well-gauged areas? A comparison with in situ observed soil moisture in an assimilation application, Adv. Water Resour., 44, 49-65, doi:10.1016/j.advwatres.2012.03.022, 2012.

McCabe, M. F., Wood, E. F., and Gao, H.: Initial soil moisture retrievals from AMSR-E: Multiscale comparison using in situ data and rainfall patterns overs Iowa, Geophys. Res. Lett., 32, 1-4, doi:10.1029/2004GL021222, 2005.

Pan, M. and Wood, E. F.: Data Assimilation for Estimating the Terrestrial Water Budget Using a Constrained Ensemble Kalman Filter, J. Hydrometeorol., 7, 534-547, doi:10.1175/JHM495.1, 2006.

Pan, M., Li, H., and Wood, E.: Assessing the skill of satellite-based precipitation estimates in hydrologic applications, Water Resour. Res., 46, W09535, doi:10.1029/2009WR008290, 2010.

Pan, M., Sahoo, A. K., and Wood, E. F.: Improving soil moisture retrievals from a physically-based radiative transfer model, Remote Sens. Environ., 140, 130-140, doi:10.1016/j.rse.2013.08.020, 2014.

Pellarin, T., Ali, A., Chopin, F., Jobard, I., and Bergès, J. C.: Using spaceborne surface soil moisture to constrain satellite precipitation estimates over West Africa, Geophys. Res. Lett., 35, 3-7, doi:10.1029/2007GL032243, 2008.

Pellarin, T., Louvet, S., Gruhier, C., Quantin, G., and Legout, C.: A simple and effective method for correcting soil moisture and precipitation estimates using AMSR-E measurements, Remote Sens. Environ., 136, 28-36, doi:10.1016/j.rse.2013.04.011, 2013.

Robock, A., Luo, L., Wood, E. F., Wen, F., Mitchell, K., Houser, P., Schaake, J., Lohmann, D., Cosgrove, B., Sheffield, J., Duan, Q., Higgins, W., Pinker, R., Tarpley, D., Basara, J., and Crawford, K.: Evaluation of the North American Land Data Assimilation System over the southern Great Plains during the warm season, J. Geophys. Res., 108, 8846, doi:10.1029/2002JD003245, 2003.

Schaake, J. C., Duan, Q., Koren, V., Mitchell, K. E., Houser, P. R., Wood, E. F., Robock, A., Lettenmaier, D. P., Lohmann, D., Cosgrove, B., Sheffield, J., Luo, L., Higgins, R. W., Pinker, R. T., and Tarpley, J. D.: An intercomparison of soil moisture fields in the North American Land Data Assimilation System (NLDAS), J. Geophys. Res.-Atmos., 109, D01S90, doi:10.1029/2002JD003309, 2004.

Schamm, K., Ziese, M., Becker, A., Finger, P., Meyer-Christoffer, A., Schneider, U., Schröder, M., and Stender, P.: Global gridded precipitation over land: a description of the new GPCC First Guess Daily product, Earth Syst. Sci. Data, 6, 49-60, doi:10.5194/essd-6-49-2014, 2014. 
Sorooshian, S.: Commentary-GEWEX (Global Energy and Water Cycle Experiment) at the 2004 Joint Scientific Committee Meeting, GEWEX Newsl., 14, 2, 2004.

Wanders, N., Pan, M., and Wood, E. F.: Correction of real-time satellite precipitation with multi-sensor satellite observations of land surface variables, Remote Sens. Environ., 160, 206-221, doi:10.1016/j.rse.2015.01.016, 2015.
Wu, H., Adler, R. F., Tian, Y., Huffman, G. J., Li, H., and Wang, J.: Real-time global flood estimation using satellite-based precipitation and a coupled land surface and routing model. Water Resour. Res., 50, 2693-2717, doi:10.1002/2013WR014710, 2014. 